\title{
Friedensprozess und Verfassungsentwicklung im Sudan ${ }^{1}$
}

\author{
Von Hatem Elliesie, Heidelberg
}

\section{Einleitung}

Abgesehen von einem 11-jährigen Zeitfenster von 1972-1983 befand sich der Sudan seit seiner Unabhängigkeit im Jahre 1956 kontinuierlich im Bürgerkrieg. Damit ist der sudanische Bürgerkrieg einer der am längsten andauernden kriegerischen, postkolonialen Auseinandersetzungen auf dem afrikanischen Kontinent. Die Ursachen hierfür sind vielfältig, lagen aber vor allem an diversen, oft miteinander verwobenen, ethnischen, religiösen, wirtschaftlichen und geostrategischen Faktoren. ${ }^{2}$ Neben religiös untermauerten, ideologischen Diskrepanzen ${ }^{3}$ stehen in diesem Zusammenhang immer mehr Fragen der Kontrolle und Förderung natürlicher Ressourcen, wie das Nilwasser und Erdölvorkommen im Süden des Landes ${ }^{4}$, im Mittelpunkt des Konflikts. Umso erfreulicher ist es für das bürgerkriegsge-

Überarbeitete Fassung des entsprechend bezeichneten Vortrags auf dem rechtswissenschaftlichen Forum des 29. Deutschen Orientalistentages an der Martin-Luther-Universität Halle-Wittenberg in Halle (Saale) am 21. September 2004.

Ähnlich auch Hanspeter Mattes, Sudan, in: Udo Steinbach [Hrsg.], Politisches Lexikon - Nahost/ Nordafrika, München 1994 (3. Auflage), S. 242 f.; er führt in diesem Bezug drei Komponenten auf: „Die arabisch islamische Identität, primär der Nordsudaner; die Nachwirkungen der Kolonialzeit sowie den religiös, ethnischen und ökonomischen Eigencharakter des Südsudan." Vgl. auch ders., Sudan Sommer 2004: Frieden im Süden, Krieg im Westen, Konflikt mit der Opposition, in: Nord-Süd aktuell, 18/2 Hamburg (August-September) 2004, S. 276 (277 f.); Rainer Tetzlaff, Bürgerkrieg und Staatsbildung im Sudan: Ein Land auf der Suche nach seiner kulturellen Identität, in: Markus Wauschkuhn / Karl Wohlmuth [Hrsg.], Die Sudanforschung in der Bundesrepublik Deutschland, München/Hamburg, 1995, S. 383 (389), ders., Ethnische Konflikte im Sudan, in: Hanspeter Mattes / Sigrid Faath [Hrsg.], Wuquf 7-8, Hamburg 1993, S. 149 (151).

Yusuf Fadl Hasan, The Role of Religion in the North-South Conflict with special Reference to Islam, in: Yusuf Fadl Hasan / Richard Gray [Hrsg.], Religion and Conflict in Sudan, Nairobi 2002, S. 23; Amir H. Idris, Sudan's Civil War - Slavery, Race and Formational Identities, African Studies 56, New York 2001, S. 77 - 104; Mansour Khalid, War and Peace in Sudan, London / New York / Bahrain 2003, S. 326 - 336, 343, 467; Francis Mading Deng, War of Visions: Conflict of Identities in the Sudan, The Brookings Institute, Washington D.C. 1995, S. 446.

Detaillierte Ausführungen dazu von Paul Goldsmith / Lydia A. Abura / Jason Switzer, Oil and Water in Sudan, in: Jeremy Lind / Kathryn Sturman [Hrsg.], Scarcity and Surfeit - The Ecology of Africa's Conflicts, Pretoria 2002, S. 187 ff.; Douglas H. Johnson, The Root Causes of Sudan's Civil Wars, Oxford 2003, S. 151; Theo Riedke, Erdölförderung verschärft den Bürgerkrieg, DEDBrief, Bonn 2000, S. 28, Ulrich Delius, Öl heizt den Krieg im Sudan an, Pogrom (210), Göttingen 2001, S. 16 f.; David Lomeling, Ewiger Kampf um Wasser und Öl, Pogrom (195/196), Göttingen 
schundene Land, dass nach mühsamen mehrjährigen Verhandlungen nun am 9. Januar 2005 ein Friedensabkommen durch die beiden Verhandlungsführer, Vizepräsident 'Alī 'Utmān Muhammad Ṭaha und dem Vorsitzenden der Sudanese People's Liberation Movement (SPLM), Dr. John Garang de Mabior, unterzeichnet wurde. Dieses Abkommen beendet zumindest den bisher maßgeblichen Nord-Süd-Bürgerkrieg in formeller Hinsicht und stellt einen weiteren essentiellen Meilenstein auf dem Weg in eine hoffungsvollere Zukunft dar. 5

\section{Der Friedensprozess}

Hoffnungsvoller auch deshalb, weil bereits in der Vergangenheit zahlreiche Bemühungen scheiterten. So hielt ein unter Vermittlung von Kaiser Haile Selassie (Hāyla Śellāsē) von Äthiopien 1972 in Addis Abeba unterzeichnete Friedensabkommen zwischen der damaligen Regierung und der Southern Sudan Liberation Movement (SSLM), mit dem der Bürgerkrieg im Südsudan durch Zugeständnisse weitgehender Autonomie an die drei Südprovinzen Äquatoria (al-’Istwā'̄ia), Baḥr al-Ġazāl und Oberer Nil (derzeit 'A`ālī an-Nīl) beendet wurden ${ }^{6}$, nur bis 1983 .

Weiterhin bemühten sich seither zahlreiche ausländische Persönlichkeiten um eine Beilegung des Südsudan-Konflikts. Nicht zuletzt wegen der Konnexität zwischen Hunger, Vertreibung und Krieg engagierten sich neben den Staatschefs Kenias, Zimbabwes, Ägyptens, Zaires, Äthiopiens und Libyens unter anderem auch der anglikanische Erzbischof von Kapstadt, Desmond Tutu, und hochrangige Vertreter christlicher und islamischer Organisationen. Auch der ehemalige US-Präsident Carter, der sich bereits seit 1986 für eine Vermittlung einsetzte, verhandelte mehrfach mit dem derzeitigen Staatspräsidenten, Stabsgeneralleutnant 'Umar Hasan Ahmad al-Bašīr, und dem SPLA-Führer Dr. John Garang de Mabior $^{7}$. Ein von Jimmy Carter im Dezember 1989 in der kenianischen Hauptstadt Nairobi arrangiertes Treffen zwischen al-Bašīr und Garang verlief jedoch enttäuschend. ${ }^{8}$

1997, S. 36 f.; siehe auch Human Development Report 2004, Chapter 3, S. 65 sowie Harker Report, Ottawa 2000.

Wegen der besonderen Eigendynamik der Entwicklungen im Sudan, kann dieser Beitrag nur eine aktuelle Bestandsaufnahme darstellen. Diese beschränkt sich auf das unmittelbare Nord-Süd Verhältnis, im Sinne der nordsudanischen Zentralregierung(en) zu den südlichen Landesteil(en), und erfasst die Geschehnisse bis zur Unterzeichnung des o.g. Friedensabkommens (Sudan's Comprehensive Peace Agreement) am 9. Januar 2005. Damit erfasst diese Abhandlung die Konflikte in Dārfūr und im Ostsudan ebenso wenig, wie konkrete Ausführungen zur Koka Dam Declaration vom März 1986 sowie zur Asmara Declaration vom Juni 1995. Addis Ababa Agreement on the Problem of Southern Sudan, Art. 3 (iii) iVm. Art. 4. 
Zudem gab es mehrere parallel laufende hoffnungsvolle Vermittlungsbemühungen, die allesamt aber nicht zu greifbaren Ergebnissen führten. ${ }^{9}$

\section{Die Intergovernmental Authority on Development}

Der gegenwärtige sudanische Friedensprozess im Allgemeinen und das nunmehr unterzeichnete Friedensabkommen im Speziellen basieren auf der Initiative der seit 1996 bestehenden Intergovernmental Authority on Development (IGAD), welche sich seit circa einem Jahrzehnt um einen substantiellen Fortschritt im Einsatz gegen diesen über vierzig Jahre dauernden Konflikt zwischen der Zentralregierung im Norden und der Widerstandsbewegung im Süden bemüht. ${ }^{10}$ Die Aufgabe der Intergovernmental Authority on Development, deren Vorläufer die 1986 ursprünglich zur Bekämpfung von Dürren und Hungerskatastrophen von sechs ostafrikanischen Staaten ${ }^{11}$ ins Leben gerufen Intergovernmental Authority on Drought and Development (IGADD) war, besteht zunehmend in der Eindämmung und Lösung der regional politischen Konflikte am Horn von Afrika 12 .

\section{Bürgenstock-Abkommen (Nuba Mountain Cease Fire Agreement)}

In der Retrospektive war die wohl entscheidende Grundlage für ergiebige Friedensverhandlungen das zwischen der sudanischen Regierung und der SPLM/Nuba in der Schweiz geschlossene Bürgenstock-Abkommen vom 19. Januar 2002, welches das vorherige Waffenstillstandsabkommen der Protagonisten im Sudan vom 13. Dezember 2001 auf die heftig

Sudanese People's Liberation Army (SPLA). Vgl. Amir H. Idris, a.a.O. (Fn. 3), S. 122; Douglas H. Johnson, a.a.O. (Fn. 4), S. 61 ff. mit weiteren Verweisen.

Die offizielle Stellungnahme Jimmy Carters, dem 39. Präsidenten der Vereinigten Staaten (19771981), ist in: Steven Wöndu / Ann Lesch, Battle for Peace in Sudan. An Analysis of the Abuja Conferences 1992 - 1993, New York / Oxford / Lanham 2000, Appendix E, S. 223 - 225, abgedruckt.

9

Wolfgang Heinrich, Der Sudan nach Machakos, in: Blätter für deutsche und internationale Politik 47, Bonn (September) 2002, S. 1051; Yusuf Fadl Hasan, a.a.O. (Fn. 3), S. 45; Ulrich Delius, Der Konflikt im Südsudan - keine Hoffnung auf Frieden, in: Hanspeter Mattes / Sigrid Faath [Hrsg.], Wuquf 7-8, Hamburg 1993, S. 189 (193).

10 Siehe: IGAD(D)-Declaration of Principles zwischen der GoS (Government of the Republic of Sudan) und der SPLM/SPLA (Sudan Peoples' Liberation Movement / Sudan Peoples' Liberation Army) in Nairobi am 20. Juli 1994.

11 Am sog. Horn von Afrika (namentlich: Äthiopien, Djibuti, Kenia, Somalia, Sudan, Uganda und zusätzlich ab 1993 auch Eritrea).

12 Vornehmlich im Sudan und in Somalia. Ausführlich zu den Entwicklungen im Sudan vgl. Abdelwahab El-Affendi, The Impasse in the IGAD Peace Process for Sudan: The Limits of Regional Peacemaking?, African Affairs 100, Oxford 2001, S. 81 f.; Zu Somalia siehe Heinrich Scholler, Somalias langer Weg zur neuen Staatlichkeit; Recht in Afrika, 2/2004, Köln 2004, S. 229 - 241. 
umkämpften zentralsudanischen Nubaberge ${ }^{13}$ ausweitete $^{14}$. Das Bürgenstock-Abkommen kam aufgrund schweizerischer und US-amerikanischer Vermittlung zustande und ebnete den Weg für Verhandlungen, indem man sich auf einen zunächst sechsmonatigen Waffenstillstand ${ }^{15}$ verständigte. ${ }^{16}$ Damit wurde der Rahmen für die Aufnahme erneuter Verhandlungen in Kenia unter der Ägide der IGAD geschaffen, was letztlich zur Unterzeichnung eines richtungweisenden Rahmenabkommens führte.

\section{Das Machakos- Protokoll}

Das am 20.Juli 2002 nach fünfwöchigen Verhandlungen von Regierung und SPLA im kenianischen Machakos unterzeichnete Protokoll ist noch kein Friedensabkommen, sondern eine breit angelegte Rahmenvereinbarung und demgemäß als Ausgangsplattform für weitere Verhandlungen zu verstehen. Dies ergibt sich schon aus der gemeinsamen Erklärung der beiden Delegationsleiter, die das gemeinhin als Machakos Protocol bekannte Dokument ${ }^{17}$ als „,breites Rahmenabkommen“ über „Prinzipien der Regierungsführung“ bezeichnen. ${ }^{18}$ Folglich legt es inhaltlich die wesentlichen Prinzipien für den künftigen staatsrechtlichen Rahmen fest und beschränkt sich in dieser Funktion dementsprechend auf die fundamentalen Grundsätze.

\section{Die Verfassungsentwicklung}

\section{Der Inhalt des Machakos-Protokolls}

Gleich zu Beginn des Machakos-Protokolls wird der Bevölkerung des Südsudans ausdrücklich das Recht auf Selbstbestimmung zugestanden, inter alia durch ein vorgesehe-

Die u.a. für die Erdölförderung strategisch wichtigen Nubaberge erstrecken sich über eine Fläche von etwa 50.000 Quadratmeilen und sind seit 1985 Kampfzone des Konflikts der beiden Opponenten. Vgl. Douglas H. Johnson, a.a.O. (Fn. 4), S. 131 - 135; Hunud Abia Kadouf, Religion and Conflict in the Nuba Mountains, in: Yusuf Fadl Hasan / Richard Gray [Hrsg.], Religion and Conflict in Sudan, Nairobi 2002, S. 107 ff.; Neroun Phillip A. Kuku, Überleben auf des Messers Schneide - Internationale Gemeinschaft muss Genozid an den Nuba stoppen, Pogrom (195/196), Göttingen 1997, S. 50 f..

15

16

17

18

Nuba Mountains Cease Fire Agreement, Preamble, S. 1.

Nuba Mountains Cease Fire Agreement, Article 1.

Regelmäßig um weitere sechs Monate verlängert, hat diese Übereinkunft bis heute weitgehend Bestand gehabt.

Presseveröffentlichung des IGAD-Sekretariats vom 23. Juli 2002.

So auch Wolfgang Heinrich, a.a.O. (Fn. 9), S. 1051 (1053); Hanspeter Mattes, Sudan 2002, in: Nahost-Jahrbuch 2002, Opladen 2004, S. 157 (162). 
nes Referendum, um den zukünftigen Status zu bestimmen. ${ }^{19}$ Darüber hinaus wurde festgelegt, dass die Parteien ein demokratisches System schaffen, das der kulturellen, ethnischen, religiösen und linguistischen Vielfalt der sudanischen Bevölkerung ebenso wie der Gleichheit der Geschlechter Rechnung tragen wird. ${ }^{20}$ Schließlich wurde als Ziel proklamiert, ein Friedenabkommen zu konzipieren und zu implementieren, wobei ausdrücklich die nationale Einheit des Sudans eine attraktive Option für das südsudanische Volk darstellen soll. $^{21}$ Dieses Ziel vor Augen wurde ein Zeitplan bis zum endgültigen Referendum im Südsudan festgesetzt. Nach Beendigung der Friedensverhandlungen und der Unterzeichnung des nun geschlossenen Friedensabkommens, soll sich eine 6-monatige Pre-Interim Period anschließen. ${ }^{22}$ Für diese erste Phase ist zunächst ganz generell die Schaffung eines verfassungsrechtlichen Rahmens vorgesehen. ${ }^{23}$ Außerdem einigte man sich darauf, in dieser Phase möglichst auch internationale Unterstützung ${ }^{24}$ heranzuziehen. ${ }^{25}$

Nach Ablauf der Pre-Interim Period beginnt die eigentliche Interim Period, welche sechs Jahre andauert, bevor letztlich ein Referendum stattfindet. ${ }^{26}$ Nach Ablauf der ersten drei Jahre ist dann eine Evaluation durch eine unabhängige Kommission vorgesehen, welche bereits in der Pre-Interim Period geschaffen wird, um in der Folgephase die Implementierung des Friedensabkommens zu überwachen. Diese sog. Independent Assessment and Evaluation Commission soll gleichermaßen aus Repräsentanten der Regierung und der SPLM zusammengesetzt sein sowie aus maximal zwei Vertretern von Mitgliedstaaten des IGAD Sub-Committees on Sudan ${ }^{27}$, Observer States ${ }^{28}$ und, nach vorheriger Vereinbarung der Parteien, aus anderen Staaten, regionalen- oder internationalen Organisationen. ${ }^{29}$

Machakos-Protocol, Part A (Agreed Principles), 1.3.

20 Machakos-Protocol, Part A (Agreed Principles), 1.6.

21

Machakos-Protocol, Part A (Agreed Principles), 1.10 sowie 1.11.

22

Machakos-Protocol, Part B (The Transition Process), 2.

Machakos-Protocol, Part A (Agreed Principles), 2.1 (f) iVm. 2.1 (a).

Das Max-Planck-Institut für ausländisches öffentliches Recht und Völkerrecht hat unter Leitung des Direktors Prof. Dr. Dr. h.c. Rüdiger Wolfrum bemerkenswerterweise bereits schon vom 18.11. bis 1.12.2002 mit hochrangigen Vertretern beider Parteien unter Beteiligung einer internationalen Expertengruppe in Heidelberg einen Draft Legal and Constitutional Framework for the Interim Period ausgearbeitet, wobei ein zu großen Teilen konsolidierter Text formuliert werden konnte. Offene politische Streitfragen wurden allerdings nicht in den Text aufgenommen, sondern der Klärung durch den entscheidenden IGAD Prozess überlassen.

Machakos-Protocol, Part B (The Transition Process), 2.1 (e).

Machakos-Protocol, Part B (The Transition Process), 2.2.

Djibuti, Eritrea, Äthiopien, Kenia und Uganda.

28 Italien, Norwegen, Großbritannien und die Vereinigten Staaten von Amerika.

29

Machakos-Protocol, Part B (The Transition Process), 2.4. Im Übrigen wird auch in diesem Teilabschnitt des Protokolls unter Ziffer 2.4.2 im zweiten Halbsatz erneut bekräftigt, dass in der 
Schließlich wird dann die Volksabstimmung nach weiteren drei Jahren unter internationaler Aufsicht gemeinsam von der Zentralregierung und der SPLM für die südsudanische Bevölkerung organisiert, in der sie entweder das aus den Friedensverhandlungen entwickelte System und damit die staatliche Einheit annehmen, oder sich für eine Abspaltung entscheiden können. ${ }^{30}$

Unter explizierter Achtung der Gewaltenteilung vereinbarte man im letzten Teilabschnitt des Protokolls ${ }^{31}$ rudimentäre Grundlagen staatlicher Strukturen und Funktionen. Die künftige (vorläufige) nationale Verfassung soll demnach unter anderem das Verhältnis zwischen den verschiedenen staatlichen Ebenen regeln und ihnen jeweilige Befugnisse und Funktionen zuweisen. Auch sollen alle Gesetze im Einklang mit dieser nationalen Verfassung stehen $^{32}$; sie soll die Grundsätze des Rechtsstaatsprinzips verwirklichen. Die Verfassung hat Vorrang vor dem einfachen Gesetz, und ihre Prinzipien sind auch für die Verfassung der territorialen Einheit verbindlich. Es gilt der Vorrang des Gesetzes; die Gewaltenteilung sowie Grundrechte und Freiheiten des Einzelnen werden garantiert. ${ }^{33}$ Die Quelle der Gesetzgebung für auf nationaler Ebene erlassene Gesetze soll die šaric $a$ (Sharia) sein, wobei sich deren Geltung nur auf Gliedstaaten außerhalb des Südsudans bezieht. ${ }^{34}$ Für den Südsudan soll gleichsam die Quelle der Gesetzgebung der popular consensus sein. Ebenso sollen dort aber auch die sittlichen Werte und Bräuche, insbesondere die jeweils vorherrschenden Gewohnheitsrechte und religiösen Überzeugungen als Äquivalent fungieren. ${ }^{35}$ In diesem Sinne soll ferner die Religionsfreiheit, die Andacht sowie die Religionsausübung allen sudanischen Bürgern uneingeschränkt verfassungsrechtlich garantiert werden. ${ }^{36} \mathrm{Um}$ für die eigentliche Interimperiode einen einfachgesetzlichen und verfassungsrechtlichen Rahmen zu entwerfen, soll in der Übergangsphase ${ }^{37}$ zwischen Unterzeichnung des Friedensabkommens und der sechsmonatigen Pre-Interim Period eine weitere Kommission

Übergangsperiode vereint darauf hingearbeitet werden soll, die nationale Einheit des Sudans als ein attraktive Option für das südsudanische Volk zu gestalten. Machakos-Protocol, Part B (The Transition Process), 2.5.

Nach der offiziellen Fassung des IGAD-Sekretariats vom 20. Juli 2002 (veröffentlicht am 23. Juli 2002) ist dies Teilabschnitt C.

Machakos-Protocol, Part C (Structures of the Government), 3.1.1.

Rüdiger Wolfrum, Föderalismus als Beitrag zur Friedenssicherung: Überlegungen zu einer Verfassung für Zypern und den Sudan, in: Michael Brenner / Peter M. Huber / Markus Möstl, Der Staat des Grundgesetzes - Kontinuität und Wandel (Festschrift für Peter Badura zum siebzigsten Geburtstag), Tübingen 2004, S. 1245 (1260).

Machakos-Protocol, Part C (Structures of the Government), 3.2.2. Weitere Ausführungen folgen!

Machakos-Protocol, Part C (Structures of the Government), 3.2.3 ff..

Machakos-Protocol, Part C (Structures of the Government), 3.1.1.

In systematischer Anlehnung an Part B (The Transition Process) des Machakos-Protokolls, wird diese Phase als Pre-Transition Period bezeichnet. 
geschaffen werden. Dieser sog. National Constitutional Review Commission kommt zudem die verheißungsvolle Aufgabe zu, das Friedensabkommen in eben diesen Rahmen zu integrieren. ${ }^{38}$

Aufgrund des besonderen Charakters als Rahmenvereinbarung, blieben trotz der Übereinkünfte zentrale Aspekte offen. Außerdem wurden mehrere Stellen des Protokolls für weitere Verhandlungen im Sinne einer constructive ambiguity ausgestaltet. So fehlen zum Beispiel Festschreibungen der territorialen Abgrenzung des Südsudans ebenso wie Aussagen über einen Modus zur Verteilung der ökonomischen Ressourcen des Landes und damit auch die Verfügung über die Einnahmen aus dem Ölexport. Entscheidende Schwachstelle war jedoch, dass im Protokoll keine Vereinbarungen über die Sicherheitsvorkehrungen während der Übergangsphase getroffen wurden. Letztlich sind nämlich frühere Abkommen im Sudan überwiegend an der unzureichenden Regelung dieser Frage gescheitert.

Das Machakos-Protokoll ebnete dennoch - oder vielleicht auch gerade deswegen - den Weg für ein als ,historisch“ bezeichnetes Treffen zwischen Präsident al-Bašīr und SPLM/A Führer Dr. John Garang de Mabior. Hauptgegenstand der Verhandlungen sollten die Aspekte power sharing und wealth sharing sein, doch blockierten andere Themen ${ }^{39}$ den Gesprächsverlauf. $^{40}$

\section{Das Memorandum of Understanding on Aspects of Structures of Government}

Nach mehreren Verhandlungsrunden wurde am 18. November 2002 in Nairobi das Memorandum of Understanding on Aspects of Structures of Government unterzeichnet, das einige der offenen Fragen in allgemeiner Form klärte, darunter „Einhaltung eines kollegialen Entscheidungsprozesses innerhalb der Präsidentschaft ““1 ${ }^{41}$, die „Bildung einer Regierung der nationalen Einheit“, die ,angemessene Berücksichtigung von Südsudaner in Exekutive

Machakos-Protocol, Part C (Structures of Government), 3.1.2. Allerdings bedarf es nach 3.1.3 letztlich der einvernehmlichen Annahme der Parteien.

39

U.a. Forderung der Regierung nach Waffenstillstand vor der Fortsetzung konkreter Gespräche, wohingegen die SPLA für einen Waffenstillstand nach erfolgreichem Verhandlungsabschluss eintrat.

Hanspeter Mattes, Sudan 2002, a.a.O. (Fn. 18), S. 162, ders. Sudan Sommer 2004: Frieden im Süden, Krieg im Westen, Konflikt mit der Opposition, a.a.O. (Fn. 2), S. 283.

41

Dem Regierungsvorschlag, John Garang zum Vizepräsidenten zu ernennen, stand seitens der SPLM der Typus einer rotierenden Präsidentschaft gegenüber. 
und Administration“, die „Einrichtung eines Zweikammerparlaments“ oder die „Verpflichtung zur Teilung von Einnahmen und Ressourcen“. 42

\section{Die Naivasha Protokolle}

Im September 2003 begannen dann direkte Verhandlungen zwischen dem sudanischen Vizepräsident Taha und dem SPLM/A Führer Garang im kenianischen Naivasha. Dabei wurden zunächst ein Teilabkommen zu Sicherheitsfragen ${ }^{43}$ und ein Abkommen zur Aufteilung der staatlichen Ressourcen sowie der Staatseinkünfte, das Wealth Sharing Agreement, unterzeichnet $^{44}$. Ein weiterer wichtiger Schritt war die Unterzeichnung dreier Teilabkommen am 26.05.2004 zur Verteilung der politischen Macht sowie zum Status der Gebiete Nubaberge, Southern Blue Nile und Abyei im Grenzgebiet zwischen Norden und Süden. Alle diese Vereinbarungen werden auch als die „Naivasha-Protokolle“ bezeichnet.

Für die konkrete Verfassungsentwicklung ist vor allem das Power Sharing Agreement von Bedeutung. Aus diesem Grund beschränkt sich der Blick im Folgenden auf dessen wesentliche Gesichtspunkte zum künftig geplanten System der Gewaltenteilung in Exekutive, Judikative und der Legislative. Das Power Sharing Agreement bezieht sich ausdrücklich auf das Machakos-Protokoll - konkretisiert es gewissermaßen in einigen Teilbereichen und bildet einen integralen Bestandteil der Friedensverhandlungen zwischen der SPLM und der sudanischen Regierung.

\section{a. Die Exekutive}

aa. Kompetenzverteilung

Der sudanischen Exekutive sollen auf nationaler Ebene der Präsident, zwei Vizepräsidenten sowie ein Ministerrat vorstehen. ${ }^{47}$ Der gegenwärtige Amtsinhaber al-Bašǐr (oder sein Nachfolger) ist als Präsident und Commander-in-Chief für die Sudan Armed Forces vorgesehen.

Hanspeter Mattes, Sudan 2002, a.a.O. (Fn. 18), S. 163, ders. Sudan Sommer 2004: Frieden im Süden, Krieg im Westen, Konflikt mit der Opposition, a.a.O. (Fn. 2), S. 283. Agreement on Security Arrangements during the Interim Period (unterzeichnet am 25. September 2003).

44 Agreement on Wealth Sharing during the Pre-Interim Period and Interim Period (unterzeichnet am 7. Januar 2004). Das Power Sharing Agreement, die Resolution of Conflict in Southern Kordofan / Nuba Mountains and Blue Nile States sowie die Resolution of Abyei Conflict.

46 Power Sharing Agreement, Part I (General Principles), 1.1 bis 1.3.

47

Power Sharing Agreement, Part II (Institutions at the National Level), 2.3.1 und 2.3.2. 
Der Vorsitzende der SPLM, Dr. John Garang de Mabior (oder sein Nachfolger) wird Erster Vizepräsident und hat zugleich das Amt des Präsidenten der südsudanesichen Regierung sowie des Commander-in-Chief der SPLA inne. ${ }^{48}$ Ihm werden für bestimmte Bereiche nach Maßgabe eines Zuständigkeitskatalogs Vetorechte gegenüber dem Präsidenten eingeräumt. ${ }^{49}$ Der Zweite Vizepräsident wird demgegenüber aller Voraussicht nach eine äußerst schwache Stellung im Vergleich zum Ersten Vizepräsidenten besitzen. Für diesen Posten ist der Vizepräsident der gegenwärtigen Regierung in Khartum und Verhandlungsführer Taha vorgesehen, der damit wohl angemessen berücksichtigt wurde.

Grundsätzlich soll der Präsident künftig gewählt werden. Der dann durch Wahlen legitimierte Präsident wird zwei Vizepräsidenten ernennen, wobei einer aus dem Norden und der andere aus dem Süden stammen sollte. Im Falle, dass aus den nationalen Präsidentschaftswahlen ein Kandidat aus dem Süden hervorgeht, ist das Amt des Ersten Vizepräsidenten mit einer Persönlichkeit aus dem Norden zu besetzen oder im umgekehrten Fall mit einem Vertreter aus dem Süden. ${ }^{50}$

Die Minister sind für Handlungen in der Ausübung ihres Amtes dem Präsidenten und der Nationalversammlung verantwortlich.

Ihres Amtes können sie mittels eines Beschlusses, der durch eine Zweidrittel-Mehrheit aller Mitglieder der Nationalversammlung getragen wird, enthoben werden. ${ }^{51}$

\section{bb. Dezentralisierte Regierungsstruktur}

Die aus dem Präsidenten, den beiden Vizepräsidenten und dem Ministerrat bestehende nationale Regierung stellt die Spitze des künftigen Staatsaufbaus dar.

Dieser ersten Ebene soll in den südlichen Landesteilen die Regierung des Südsudans zunächst mit dem nationalen ersten Vizepräsidenten als Präsident folgen. ${ }^{52}$ Sie soll als Verbindungsglied zwischen der nationalen Regierung und den Gliedstaaten im Süden nach Maßgabe der nationalen Übergangsverfassung und der künftigen südsudanischen Verfassung fungieren. ${ }^{53}$ In Konsultation mit dem/der Vizepräsidenten/in des Südsudans soll nach

48

49

50

51

52

53

Power Sharing Agreement, Part II (Institutions at the National Level), 2.3.5.

Power Sharing Agreement, Part II (Institutions at the National Level), 2.3.6.

Power Sharing Agreement, Part II (Institutions at the National Level), 2.3.7.

Power Sharing Agreement, Part II (Institutions at the National Level), 2.3.12 (Satz 2).

Power Sharing Agreement, Part II (Institutions at the National Level), 2.3.5 (Satz 2).

Vgl. Power Sharing Agreement, Part I (General Principles), 1.5.1.3. 
Zustimmung des südsudanischen Parlaments ein Ministerrat, der so genannte Executive Council of Ministers, durch den südsudanischen Präsidenten ernannt werden. ${ }^{54}$

Obwohl im Rahmen der Amtshandlungen nach dem genauen Wortlaut 3.6.1 des Power Sharing Agreements ${ }^{55}$ nicht der einzelne Minister, sondern der Ministerrat als Gremium (Executive Council of Ministers) gegenüber der südsudanischen Versammlung verantwortlich sein soll, war hier eine der nationalen Ebene identische Regelung beabsichtigt. ${ }^{56}$

Für die Amtsenthebung der Minister ist auch hier eine entsprechende Zweidrittel-Mehrheit aller Mitglieder des Executive Council of Ministers erforderlich. ${ }^{57}$

Im Staatsaufbau folgen dann die Gliedsstaaten des Südens mit den Local Governments, die wiederum in Counties untergliedert sind. Als administrative Untereinheiten der Counties folgen dann die Payams und/oder Stadträte (Town Councils) und letztlich, als kleinste Einheit in diesem Sinne, die Bomas. .

Erstaunlich ist im Hinblick auf den Norden, dass dort bisher offenbar kein dezidiertes Äquivalent zur südsudanischen Regierung geregelt ist. Ob diese Ebene im Staatsaufbau vorerst gänzlich entfällt, und damit die Gliedstaaten unmittelbar der nationalen Regierung unterstellt werden, sie also eine Doppelfunktion wahrnimmt ${ }^{58}$ oder marginale personelle Änderungen vorgenommen werden oder aber, ob die derzeitigen Machthaber in Khartum stillschweigend davon ausgehen, dass ihre bisherigen Positionen weitgehend in einer eigenständigen nordsudanischen Regierung fortbestehen, bleibt abzuwarten.

The Executive Council of Ministers shall be accountable to the President of the Government of Southern Sudan and the Southern Sudan Assembly in the performance of their functions and may be removed by a motion supported by two-thirds of all the members of the Southern Sudan Assembly.

Dies ergibt sich aus dem weiterführenden Satz durch die Verwendung des Possessivpronomens ,their" und nicht ,its“. Damit wird deutlich, dass nicht die Körperschaft, sondern der einzelne Minister gemeint ist. Dafür spricht ebenso der eindeutige Wortlaut unter Part IV (Institutions at the State Level) in 4.5.3 (Satz 2) des Power Sharing Agreements, welcher in unmittelbarem systematischem Zusammenhang mit der o.g. Regelung steht und damit diese Auffassung bekräftigt. 4.5.3 (Satz 2) des Power Sharing Agreement lautet:

The State Ministers shall be accountable to the Governor and the State Legislature in the performance of their functions and may be removed by the Governor on a motion supported by two-thirds of all the members of the State Legislature. 
Die erste Variante scheint aber kaum realistisch zu sein, würde es doch faktisch bedeuten, dass durch die gemischte nationale Regierung erheblicher südsudanischer Einfluss aufkommen würde, der die Hegemonie nordsudanischer Eliten beschneiden würde. Es ist kaum vorstellbar, dass die nicht unerheblichen politischen Kräfte des Nordens dies zulassen wollen. Abgesehen von diesem Politikum, wirft diese Alternative zusätzlich eine Reihe außergewöhnlich komplexer, verfassungsrechtlicher Fragen auf. Es ist abzusehen, dass die Rechtsordnung des Nordens weitgehend auf der Basis der šari ${ }^{`} a$ beruhen wird ${ }^{59}$, und entsprechend müsste die nationale Regierung, soweit sie als Regierung für den Norden agiert, dem Rechnung tragen. Als Bundesregierung hat sie aber nach Vorstellung der SPLM und nach bisherigen Einigungen religiös neutral zu sein. ${ }^{60}$

Gegen einen stillschweigenden Fortbestand leitender Regierungsämter im Norden wird sich andererseits nach den gegenwärtigen Entwicklungen erheblicher Widerstand aus den Reihen der Opposition stark machen. Auch sie werden bestimmt Partizipationsansprüche geltend machen.

Klar geregelt ist insoweit nur, dass der regierenden National Congress Party (NCP) und der SPLM je $70 \%$ in den jeweiligen Landesteilen bis zu den ersten Wahlen zugewiesen werden. Von den übrigen $30 \%$ hat man sich zudem gegenseitig $10 \%$ zugestanden, so dass letztlich im Norden wie im Süden $20 \%$ für andere Parteien übrig bleiben. ${ }^{61} \mathrm{Ob}$ dies von den anderen politischen Gruppierungen in dieser Form ohne weiteres hingenommen wird, mag bezweifelt werden. ${ }^{62}$

Eine Volkszählung soll bis zum Ende des zweiten Jahres der Interimperiode vollständig durchgeführt werden. Die allgemeinen Wahlen auf allen Regierungsebenen müssen dann bis zum Ende des folgenden Jahres dieser Übergangsphase stattfinden. ${ }^{63}$

\section{b. Die Judikative}

Die Übergangsverfassung soll die Unabhängigkeit der Justiz gewährleisten. Auf nationaler Ebene ist ein 4-gliedriger Justizaufbau vorgesehen. Dieser setzt sich zusammen aus dem Verfassungsgericht, einem Obersten Gerichtshof, dem National Supreme Court, den nationalen Berufungsgerichten (National Courts of Appeal) und anderen, durch Gesetz ge- 
schaffenen, nationalen Gerichten und Tribunalen, soweit diese für nötig erachtet werden. ${ }^{64}$ Im Power Sharing Agreement sind bisher nur für die beiden ersten Institutionen Regelungen getroffen worden. Auf sie beschränkt sich die Untersuchung, ehe dann auf die südsudanischen Bestimmungen zur Judikative einzugehen ist.

\section{aa. Verfassungsgericht}

Primär soll das Verfassungsgericht für aufkommende Kontroversen im Zusammenhang mit der nationalen Übergangsverfassung und der Verfassungen der nördlichen Gliedstaaten zuständig sein. Solche verfassungsrechtlichen Entscheidungen können unmittelbar durch Individualklagen, durch Klageanträge von juristischen Personen in Form von Individualbeschwerden oder auch aber auch durch die Regierung mittels Normenkontrollanträgen und im Übrigen durch Organstreitverfahren begehrt werden. ${ }^{65}$ Zudem wird dem Verfassungsgericht unter anderem die besondere strafrechtliche Gerichtsbarkeit über den nationalen Präsidenten, über seine beiden Vizepräsidenten sowie die Richter, sowohl des nationalen, als auch des südsudanischen Obersten Gerichtshofes, unterstellt. ${ }^{66}$ Die Entscheidungen des Verfassungsgerichts werden endgültig und bindend sein. ${ }^{67}$

Der Präsident des Verfassungsgerichts wird durch den Staatspräsidenten mit Zustimmung des Ersten Vizepräsidenten ernannt und hat sich nur gegenüber der Präsidentschaft zu verantworten. $^{68}$ Die übrigen Verfassungsrichter werden auf Vorschlag einer National Judicial Service Commission durch die Präsidentschaft unter Vorbehalt der Zustimmung einer Zweidrittel- Mehrheit aller Mitglieder des Rates der Gliedsstaaten ernannt. ${ }^{69}$

\section{bb. Oberster nationaler Gerichtshof}

Der National Supreme Court soll die Funktion eines Revisionsgerichtes und Kassationshofes bezüglich strafrechtlicher und zivilrechtlicher Angelegenheiten innehaben, welche sich aus oder unter den einfach gesetzlichen Nationalgesetzen ergeben. Darüber hinaus ergeben sich spezielle Zuständigkeiten, wie die strafrechtliche Gerichtsbarkeit über die nationalen Verfassungsrichter und Revisionen zu Todesstrafen, die von irgendeinem Gericht aus oder unter der einfach gesetzlichen, nationalen Normenklassifikation verhängt oder erlassen wurden. Weitere Zuständigkeiten werden noch durch die Übergangsverfas-

64

65

66

67

68

69

Power Sharing Agreement, Part II (Institutions at the National Level), 2.11.1 und 2.11.2.

Vgl. Power Sharing Agreement, Part II (Institutions at the National Level), 2.11.3.2 (iii - vi).

Power Sharing Agreement, Part II (Institutions at the National Level), 2.11.3.2 (viii).

Power Sharing Agreement, Part II (Institutions at the National Level), 2.11.3.3.

Power Sharing Agreement, Part II (Institutions at the National Level), 2.11.3.2 (i).

Power Sharing Agreement, Part II (Institutions at the National Level), 2.11.4.6 (i). 
sung, den Friedensvertrag und Gesetz bestimmt. Soweit besondere Expertise im Rahmen einer Revision erforderlich sein sollte, werden dafür spezielle Kammern gebildet. ${ }^{70}$

Die Instanzrichter selbst werden ebenso wie die Mitglieder des Verfassungsgerichts auf Vorschlag der National Judicial Service Commission von der Präsidentschaft ernannt. Allerdings bedarf es hier nicht der Zweidrittel-Mehrheit aus dem Rat der der Gliedstaaten. Ihr Amt sollen sie unabhängig und ohne politische Einmischung, Gefälligkeiten und Angst ausüben können. Dieser Vorstellung soll die Übergangsverfassung nach dem Power Sharing Agreement Rechnung tragen.

\section{cc. Das südsudanische Gerichtswesen}

Auf der südsudanischen Ebene soll einem Supreme Court of Southern Sudan, also einem oberster Gerichtshof des Südsudans, Berufungsgerichte (Courts of Appeal) sowie andere, aufgrund der südsudanischen Verfassung und durch Gesetz geschaffene, für nötig erachtete, nationale Gerichte und Tribunale folgen. ${ }^{72}$ Auch hier sind noch keine umfassenden Regelungen getroffen worden. Dementsprechend kann an dieser Stelle nur auf den obersten Gerichtshof des Südsudans eingegangen werden.

Der Supreme Court of Southern Sudan soll der letztinstanzliche Entscheidungsträger bezüglich aller Rechtsstreitigkeiten oder Anklagen unter südsudanischem Recht sein. Er ist zuständig für verfassungsrechtliche Kontroversen unter der Verfassung des Südsudans oder Verfassungen südsudanischer Gliedstaaten. Entscheidungen können auch hier unmittelbar durch Individualklagen, durch Klageanträge von juristischen Personen in Form von Individualbeschwerden oder auch aber auch durch die Regierung mittels Normenkontrollanträgen und im Übrigen durch Organstreitverfahren begehrt werden.

Daneben kommt diesem Gericht eine vergleichbare Funktion wie dem Obersten Nationalen Gerichtshof zu. Es ist ebenso ein Revisionsgericht und Kassationshof bezüglich strafrechtlicher und zivilrechtlicher Angelegenheiten, die sich aus oder unter dem einfachen südsudanischen Gesetz ergeben. Darüber hinaus sind ebenfalls Zuständigkeiten im Bezug auf Revisionen zu Todesstrafen, die von einem südsudanischen Gericht ausgesprochen oder unter den einfach gesetzlichen südsudanischen Normen verhängt wurde, vorgesehen. Der südsudanische Präsident und sein Vizepräsident haben sich vor ihm zu verantworten.

Power Sharing Agreement, Part II (Institutions at the National Level), 2.11.4.1 (i) - (iv); 2.11.4.2.

Power Sharing Agreement, Part II (Institutions at the National Level), 2.11.4.3 - 2.11.4.4; 2.11.4.6 (ii).

72

Power Sharing Agreement, Part II (Institutions at the National Level), 3.7.1. 
Dass Dr. John Garang de Mabior sowohl nationaler Vizepräsident, als auch südsudanischer Präsident in Personalunion sein wird, wurde in diesem Kontext bei den Verhandlungen offenbar nicht ausreichend in die Überlegungen miteinbezogen. Hier sollten möglichst Kriterien determiniert werden unter welchen die gerichtlichen Zuständigkeiten aufgeteilt werden. Denkbar wäre natürlich auch als Anknüpfungspunkt generell auf den Sachzusammenhang seines Handelns abzustellen. Jedenfalls ist es zweckdienlich, sich mit dieser Angelegenheit weiter auseinanderzusetzen und entsprechende Regelungen zu fixieren.

Die weiteren Zuständigkeiten werden noch durch die südsudanische Verfassung, den Friedensvertrag und das Gesetz bestimmt. ${ }^{73}$

Für die Südsudaner ergibt sich aufgrund der zusätzlichen Ebene im Staatsaufbau eine weitere Funktion. Für sie ist nämlich das Verfassungsgericht die letzte Instanz gegen verfassungsrechtliche Entscheidungen des Southern Sudan Supreme Court, welche spezifisches südsudanisches Verfassungsrecht zum Gegenstand haben. ${ }^{74}$

\section{c. Die Legislative}

Für die nationale Legislative ist ein bikamerales System vorgesehen, bestehend aus einem National Assembly, also einer Nationalversammlung und einem Rat der Gliedstaaten, dem Council of States. ${ }^{75}$ Die Mitglieder einer Nationalversammlung sollen entsprechend einem von einer unparteiischen und repräsentativen Wahlkommission gesetzlich festgelegten Verfahren gewählt werden. ${ }^{76}$ Bei der Verteilung der Sitze in der Nationalversammlung sind $52 \%$ auf die NCP, $28 \%$ auf die SPLM, $14 \%$ auf andere politische Kräfte des Nordens und $6 \%$ für sonstige politische Kräfte des Südens veranschlagt. ${ }^{77}$ Der Council of State setzt sich hingegen aus jeweils zwei Vertretern der Gliedstaaten zusammen. ${ }^{78}$ Die konkrete Zusammensetzung, der Umstand, ob die Vertreter gewählt oder ernannt werden, ist bisher allerdings noch nicht schriftlich fixiert worden.

aa. Verfassungs- und Gesetzesänderungen

Auch wenn es einen rein formellen Akt darstellt, so bedarf der nun nach Friedensschluss auszuarbeitende Verfassungstext der Annahme sowohl durch die gegenwärtige National-

Power Sharing Agreement, Part II (Institutions at the National Level), 3.7.3.1 bis 3.7.3.7.

Power Sharing Agreement, Part II (Institutions at the National Level), 2.11.3.2 (v) iVm. 2.11.3.3.

Power Sharing Agreement, Part II (Institutions at the National Level), 2.2.1.

Power Sharing Agreement, Part II (Institutions at the National Level), 2.2.3.1.

Power Sharing Agreement, Part II (Institutions at the National Level), 2.2.5.

Power Sharing Agreement, Part II (Institutions at the National Level), 2.2.3.2. 
versammlung, als auch durch den SPLM National Liberation Council, um verfassungsrechtlich das Übergangsregime bis zum Ende der Interimsperiode zu tragen. ${ }^{79}$

Für Änderungen dieser nationalen Verfassung soll es der Zustimmung von 3/4 aller Mitglieder beider Kammern bedürfen. Dabei sollen beide Kammern getrennt voneinander tagen. Änderungsanträge sind außerdem mindestens zwei Monate vor der eigentlichen Debatte einzureichen. Änderungen der Übergangsverfassung hingegen, welche Regelungen der Friedensvereinbarung betreffen, bedürfen der Zustimmung aller Parteien des Friedensabkommens. 80

Ferner ist eine 2/3 Mehrheit im Rat der Gliedstaaten erforderlich, um Gesetze zu verabschieden, die die Interessen der Gliedstaaten betreffen. Für die Verabschiedung aller anderen Gesetze soll die einfache Mehrheit in beiden Kammern ausreichen. ${ }^{81}$

\section{bb. Nationales Gesetzgebungsverfahren}

Ein vom nationalen Gesetzgeber ordnungsgemäß beschlossenes Gesetz bedarf der Unterschrift des Präsidenten innerhalb von 30 Tagen nach Beschlussfassung. Wird die Unterschrift verweigert, sind die Gründe hierfür anzugeben, wenn der Gesetzesentwurf erneut dem nationalen Gesetzgeber innerhalb der 30 Tage vorgelegt wird. Das Gesetz kommt schließlich zustande, sofern der nationale Gesetzgeber den Entwurf wiederum mit einer 2/3 Mehrheit aller Mitglieder der jeweiligen Versammlungen oder Versammlung verabschiedet. Die Zustimmung des Präsidenten soll dann nicht mehr erforderlich sein. Ein explizites Vetorecht des Präsidenten ist bisher nicht vorgesehen. ${ }^{82}$

\section{cc. Gesetzgebungskompetenzen}

Die künftige Verteilung der Gesetzgebungskompetenzen zwischen der nationalen Ebene und der regionalen Ebene im Süden lässt sich mit deutschen Vorstellungen vergleichen. Hier haben südafrikanische Verfassungsvorstellungen Pate gestanden, die mit deutschen verfassungsrechtlichen Prinzipien vergleichbar sind. ${ }^{83}$ Dies ist keineswegs verwunderlich, so gilt die Verfassungsentwicklung im „Neuen Südafrika“ als Musterbeispiel für eine

79

80

81

82

83
Power Sharing Agreement, Part II (Institutions at the National Level), 2.12.7.

Power Sharing Agreement, Part II (Institutions at the National Level), 2.2.7.1 und 2.2.7.2.

Power Sharing Agreement, Part II (Institutions at the National Level), 2.2.7.3.

Power Sharing Agreement, Part II (Institutions at the National Level), 2.2.8.

Rüdiger Wolfrum, a.a.O. (Fn. 33), S. 1262 
Rezeption deutschen Verfassungsrechts im Ausland. ${ }^{84}$ Neben einer ausschließlichen Gesetzgebungskompetenz des nationalen Gesetzgebers ${ }^{85}$ sind auch konkurrierende Gesetzgebungskompetenzen ${ }^{86}$ und spezifische Kompetenzen der Gliedstaaten, insbesondere für die südlichen Regionen, vorgesehen. ${ }^{87}$

Noch nicht richtig geklärt ist außerdem, wie das Spannungsverhältnis zwischen nationalem Recht und dem Recht der Gliedstaaten ausgestaltet sein soll. Bislang konnte man sich nur darauf einigen, dass die Regelung Vorrang haben wird, welche „,most effectively deals with the subject". Es wird sich zeigen, ob hier beispielsweise eine dem Art. 31 GG (Bundesrecht bricht Landesrecht) vergleichbare Regelung geschaffen wird oder ob man es der richterlichen Fortentwicklung überlässt.

Interessant ist hierbei aber auch, inwieweit und ob man sich an vergleichbaren Regelungen afrikanischer Staaten orientiert. Grundsätzlich soll die Verfassung Vorrang vor dem einfachen Recht haben, ihre Prinzipien sind auch für die Verfassungen der territorialen Einheit verbindlich. ${ }^{88}$ Insoweit unterscheidet sich die Konzeption nicht von den verfassungsrechtlichen Vorgaben beispielsweise gemäß Artikel 9 in Äthiopien, Artikel 2 in Uganda, Section 1 in Nigeria oder der südafrikanischen Section 2. Ob man es textlich jedoch dabei belässt, ${ }^{89}$ sich an einer prägnanten Formulierung wie in Section 4 Absatz 5 der nigerianischen Verfassung ${ }^{90}$ orientiert oder sich für eine der detaillierten südafrikanischen Regelung ${ }^{91}$ ver-

André Thomashausen, Demokratie und Rechtsstaatlichkeit: Südafrika, in: Ulrich Battis / Philip Kunig / Ingolf Pernice / Albrecht Randelzhofer [Hrsg.], Das Grundgesetz im Prozess europäischer und globaler Verfassungsentwicklung, Baden-Baden 2000, S.163. So auch Johan de Waal, A Comparative Analysis of the Provisions of the German Origin of the Interim Bill of Rights, SAJHR 11, Johannesburg / Cape Town 1995, S.1 f., der zum südafrikanischen Übergangsregime unter anderem konstatierte, dass „... it is not possible to understand the Interim Constitution itself without appreciation of the German origin ...".

Power Sharing Agreement, Part II (Institutions at the National Level), 2.2.9 iVm. Part V, Schedule A.

Power Sharing Agreement, Part II (Institutions at the National Level),2.2.10 iVm. Part V,Schedule D.

Vgl. Power Sharing Agreement, Part V, Schedule B und C.

Rüdiger Wolfrum, a.a.O. (Fn. 33), S. 1260.

So zum Beispiel in Äthiopien und Uganda.

90

Section 4 der nigerianischen Verfassung:

(5) If any Law enacted by the House of Assembly of a State is inconsistent with any law validly made by the National Assembly, the law made by the National Assembly shall prevail, and that other Law shall, to the extent of the inconsistency, be void.

Section 146 der südafrikanischen Verfassung:

(2) National legislation that applies uniformly with regard to the country as a whole prevails over provincial legislation if any of the following conditions is met: 
gleichbaren Gesetzmäßigkeit entscheidet, ist noch völlig ungewiss. Sollte sich hier eine dem südafrikanischen Modell entsprechende Lösung durchsetzen, so darf man gespannt sein, ob eine ebenso deutliche Formulierung festlegt wird, wonach die nationale Gesetzgebung selbst dann den regionalen Normen vorgeht, wenn ein Disput nicht vor einem Gericht beigelegt werden kann. ${ }^{92}$

\section{dd. Zur (verfassungsrechtlichen) Signifikanz der šari`a}

Während in Westeuropa die Verfassungen als Ergebnis des Ringens um jenen „heiligen Text" als Grundriss der staatlichen Ordnung und Machtverteilung, einen hohen normativen Wert haben, wird der normative Charakter der arabischen Verfassungen bisher vergleichsweise gering eingeschätzt. ${ }^{93}$ Aus der Tatsache heraus, dass ein Herrschaftswechsel oder systematischer Richtungswechsel die Proklamation einer neuen Verfassung ${ }^{94}$, eine Totalrevision ${ }^{95}$ oder eine partielle Modifikation ${ }^{96}$ nach sich zieht, wird gefolgert, dass die meisten

(a) The national legislation deals with a matter that cannot be regulated effectively by legislation enacted by the respective provinces individually.

(b) The national legislation deals with a matter that, to be dealt with effectively, requires uniformity across the nation, and the national legislation provides that uniformity by establishing -

(i) norms and standards;

(ii) frameworks; or

(iii) national policies.

(c) The national legislation is necessary for -

(i) the maintenance of national security;

(ii) the maintenance of economic unity;

(iii) the protection of the common market in respect of the mobility of goods, services, capital and labour;

(iv) the promotion of economic activities across provincial boundaries;

(v) the promotion of equal opportunity or equal access to government services; or

(vi) the protection of the environment.

(3) National legislation prevails over provincial legislation if the national legislation is aimed at preventing unreasonable action by a province that -

(a) is prejudicial to the economic, health or security interests of another province or the country as a whole; or

(b) impedes the implementation of national economic policy.

(5) Provincial legislation prevails over national legislation if subsection (2) or (3) does not apply.

Section 148 der südafrikanischen Verfassung:

If a dispute concerning a conflict cannot be resolved by a court, the national legislation prevails over the provincial legislation or provincial constitution.

Hanspeter Mattes, Moderne Arabische Verfassungsentwicklung, in: Hanspeter Mattes / Sigrid Faath [Hrsg.], Demokratie und Menschenrechte in Nordafrika, Hamburg 1992, S. 49 f..

So beispielsweise in Libyen im Jahre 1969; wobei Syrien mit sechs Verfassungen in einem Zeitraum von 22 Jahren bisheriger Spitzenreiter sein soll.

Tunesien im Jahre 1987. 
arabischen Verfassungen nicht für einen längeren Zeitraum normativ sind, sondern jedes neue Staatsoberhaupt eine neue Verfassung ausarbeiten lässt, die das Selbstverständnis und die Regierungsform am besten zum Ausdruck bringt. ${ }^{97}$

Diese historische Analyse - arabische Verfassungen haben weniger einen normativen, als vielmehr einen programmatischen Charakter - mag im Kern zutreffend sein, ist aber keineswegs ein Phänomen, welches sich per se auf die Mitgliedstaaten der Arabischen Liga reduzieren lässt, sondern geht mit revolutionär-politischen Umwälzungen in Entwicklungsländern im Allgemeinen einher. So hatten solche Herrschaftswechsel und systematische Richtungswechsel gegenwärtiger oder ehemals autoritär-geprägter Regime auch in anderen Regionen der Welt nicht selten ideologisch bedingte Veränderungen in der staatlichen Grundordnung zur Folge. Verfassungen sind eben nicht nur pragmatisch, sondern auch programmatisch. Mit anderen Worten: Die Verfassungen gehen über die Regelungen des Staatsorganisationsrechts hinaus und bringen eine bestimmte Axiologie zum Ausdruck. ${ }^{98}$ Dass diese machtpolitisch monopolisierten Manifeste dann für sich betrachtet das jeweilige Selbstverständnis bestimmter Eliten widerspiegelt, ist also - jedenfalls verfassungsvergleichend - im Lichte eines weiteren Kontextes zu beleuchten. Ferner besteht bei einer solchen ,,arabisch-historischen Analyse“ das Wagnis der Vermengung gemeinsamer (Religions-) Geschichte, Sprache und Religion mit faktisch heterogenen ökonomischgesellschaftlichen Wandlungen der einzelnen Staaten, welche sich damit auch in differenten Stadien gegenwärtiger Verfassungsentwicklung befinden. ${ }^{99}$

Im Rahmen der momentanen sudanischen Entwicklung wurde im Machakos-Protokoll klar zum Ausdruck gebracht, dass $\check{s ̆ a r i ~}^{`} a$ (Sharia) als Quelle der nationalen Gesetzgebung nur für Gliedstaaten außerhalb des Südsudans gelten soll. ${ }^{100}$ Es ist in diesem Zusammenhang nicht unbeachtlich, dass bislang nicht von „,der Quelle“ die Rede ist. Heißt es nämlich, die šari` $a$ sei „,die Quelle“ der Gesetzgebung, so ist ihr Einfluss generell größer als bei der Formulierung, die šari`a sei lediglich „eine Quelle“ der Gesetzgebung. ${ }^{101}$ Dass im Machakos-Protokoll die Formulierung

So Hanspeter Mattes, Moderne Arabische Verfassungsentwicklung, a.a.O. (Fn. 93), S. 49 (50). Allerdings ist nicht ganz klar, welche Art des „Herrschaftswechsels“ hier zugrunde gelegt wird. Denn beispielsweise in Ägypten folgte nämlich keine Verfassungsänderung in Folge der Amtsübernahme des neuen Präsidenten Mubārak.

Zdzislaw Kędzia, 50 Jahre Grundgesetz - Eine Inspiration, in: Ulrich Battis / Philip Kunig / Ingolf Pernice / Albrecht Randelzhofer [Hrsg.], Das Grundgesetz im Prozess europäischer und globaler Verfassungsentwicklung, Baden-Baden 2000, S. 43 (47).

Insoweit wieder zustimmend mit Hanspeter Mattes, Moderne Arabische Verfassungsentwicklung, a.a.O. (Fn. 93), S. 49 (69).

100 Machakos-Protocol, Part C (Structures of the Government), 3.2.2.

101 Hanspeter Mattes, Moderne Arabische Verfassungsentwicklung, a.a.O. (Fn. 93), S. 49 (70). 
„Nationally enacted legislation having effect only in respect of the states outside Southern Sudan shall have as its source of legislation Sharia and the consensus of the people"

unter Ziffer 3.2.2 fixiert wurde, lässt aber keineswegs den Schluss zu, dass die Formulierung rein programmatischen Charakter oder lediglich den Charakter allgemeiner Respektsüberzeugung hätte und demnach in materieller Hinsicht bedeutungslos sei. Andererseits wird diese, seit den 60er Jahren auch in anderen Verfassungen der Region vergleichbar konstituierte Formulierung ${ }^{102}$, wonach die Prinzipien der $\check{\text { šari }}{ }^{\complement} a$ eine ${ }^{103}$ Quelle der Gesetzgebung ist ${ }^{104}$, die $\check{s} a i^{\complement} a$ in diesem Sinne sicherlich nicht zu einem absolut und umfassend gültigen gesellschafts- und rechtspolitischen System erheben. Eine Beurteilung, inwieweit Verfassungen den Grundsätzen der $\check{s}{ }^{i}{ }^{\complement} a$ entsprechen, lässt sich ohnehin nicht durchführen, da die šari ${ }^{\complement} a$ keine eindeutigen Hinweise für die materielle Ausgestaltung einer Verfassung bietet. Am ehesten scheint noch die iranische Verfassung der islamischen Rechtstradition $\mathrm{zu}$ entsprechen. Allerdings ist in dieser Verfassung unter anderem das Imamatsdogma verankert, ein Element, das für sunnitisch dominierte Gebiete, also auch für den Sudan, nicht annehmbar ist. ${ }^{105}$

Grundsätzlich, aber auch speziell für die bevorstehende verfassungsmäßige Verankerung des Terminus der „Quelle der Gesetzgebung“ und dessen praktische Bedeutung wie theoretische Einordnung, sind vielmehr die sozioökonomischen, politischen, religiösen und verfassungshistorischen Faktoren ausschlaggebend. Je nachdem in welcher historischen Etappe der staatlichen Entwicklung man sich befindet, beziehen sie sich auf unterschiedliche gesellschaftliche Bedingungen und orientieren sich an unterschiedlichen Vorbildern. ${ }^{106}$

102

So u.a. die gegenwärtigen Verfassungen von Ägypten (Artikel 2), Bahrain (Artikel 2), Kuwait (Artikel 2), Syrien (Artikel 3), Oman (Artikel 2), Mauretanien (Präambel), die Vereinigten Arabi103 schen Emirate (Artikel 7) und Jemen (Artikel 3).

Zur Verfassungsänderung Ägyptens im Jahre 1980, wonach die šari` $a$ als ,eine Quelle der Gesetzgebung“ durch die Formulierung ,die Quelle der Gesetzgebung“ ersetzt wurde; vgl. Omaia Elwan, Gesetzgebung und Rechtsprechung, in: Udo Steinbach / Rüdiger Robert [Hrsg.], Der Nahe und Mittlere Osten, Opladen 1988, ausführlich zu Ägypten S. 237 ff. sowie zu der Lage in Verfassun104 gen anderer Mitgliedstaaten der Arabischen Liga S. 249 ff..

Vgl. dazu auch Baber Johansen, The Relationship between the Constitution, the Sharî'a and Fiqh: The Jurisprudence of Egypt's Supreme Constitutional Court, ZaöRV (HJIL) 64, Stuttgart 2004, S. 885 - 896; Kilian Bälz, Das moderne arabische Recht, in: Hans-Georg Ebert / Thoralf Hanstein [Hrsg.], Beiträge zum Islamischen Recht II (Leipziger Beiträge zur Orientforschung, Band 12), Frankfurt a.M. 2003, S.175 (179).

105 Omaia Elwan, Gesetzgebung und Rechtsprechung, a.a.O. (Fn. 103), S. 251.

106 Vgl. Hans-Georg Ebert, Die Interdependenz von Staat, Verfassung und Islam im Nahen und Mittleren Osten in der Gegenwart, Frankfurt a.M. 1991, S. 146 und S. 202. 


\section{Anmerkungen}

\section{Zur Verfassungsentwicklung}

Zumindest nach derzeitiger Prognose könnte diese Formulierung im Machakos-Protokoll möglicherweise ein richtungweisender Impuls im auf der Suche nach „Authenzität“ $\left(\right.$ așa $\left._{\bar{a}} a^{107}\right)$ und, oder wohl eher ein Ringen um eine eigenständige sudanische Synthese aus „,westlichen“ und „islamischen“ Rechtsvorstellungen sein. ${ }^{108}$

Wie die grundsätzlich durch die dargelegten Agreements und speziell durch die Formulierung 3.2.2 des Machakos-Protokolls beleuchtete Verfassungsentwicklung belegt, wird erneut eine verstärkte Rezeption westlicher, vom Säkularismus und Föderalismus geprägter Rechtssysteme erwogen. Dies basierend nicht zuletzt auf den erfolglosen „Reformversuch(ung)en“ der letzten Jahrzehnte.

Föderalismus ${ }^{109}$ ist bisher zwar in einer großen Anzahl der Mitgliedstaaten der Arabischen Liga, aufgrund der historisch gewachsenen starken Zentralstaatlichkeit, eine kaum bekannte oder höchstens ansatzweise feststellbare Erscheinung,und auch im afrikanischen Vergleich stellt das faktische Streben der politischen Eliten im Sudan, seit seinem Bestehen als völkerrechtlich souveräne Einheit, einen Einheitsstaat zu schmieden, keine Besonderheit dar. ${ }^{110}$ Dennoch bezeichnet die sudanische Staatsführung unter al-Bašīr seit 1991 den Sudan als föderalen Staat. Als Argument für den föderalen Charakter des Staates wird

107

Dazu Gudrun Krämer, Gottes Staat als Republik - Reflexion zeitgenössischer Muslime zu Islam, Menschenrechten und Demokratie, Baden-Baden 1999, S. 15 - 21.

108 Interessante Aspekte zum innerislamischen Diskurs finden sich u.a. im „Entwurf einer Islamischen Verfassung“ (mašrū̌c ad-dustūr al-islāmī), welcher im Jahre 1978 von der „Akademie für Islamische Studien“" (mağma` al-buhūt al-islāmiyya) an der Kairoer Azḥar-Universität verabschiedet wurde. Auch wenn dieser Entwurf der bedeutendsten Institution des sunnitischen Islam keine Verbindlichkeit für zeitgenössische sunnitische Muslime beansprucht, so verdient diese für Muslime in der ganzen Welt konzipierte Modellverfassung im akademischen Diskurs und im überseeischen Verfassungsvergleich Beachtung: In arabischer Sprache veröffentlicht in Mağallat al-Azḥar 51, Kairo 1979 (4), S. 1092 - 1100. Leicht divergierende englische Übersetzungen dieser Fassung erschienen in der englischen Sektion der Majallatu 'l-Azhar 51, Kairo 1979 (4), S. 151 - 159 (arabische Seitenzählung S. 1118 - 1126). sowie in Muslim World League Journal, April 1982, S. 29 - 34 (Übersetzung und Kommentar durch $A l-\dot{G} a z \bar{a} l \bar{\imath}$ ). Zu der in den sechziger Jahren gegründeten Akademie für islamische Studien siehe Jakob Skovgaard-Petersen, Defining Islam fort he

109 Egyptian State. Muftis and Fatwas of the Dār al-Iftā, Leiden u.a. 1997, S. 186 - 188.

Auf die vielfältigen Definitionen, Formen und Ausprägungen der Begrifflichkeit kann in diesem Beitrag nicht eingegangen werden. Vgl. dazu Ernst Deuerlein, Föderalismus - Die historischen und philosophischen Grundlagen des föderativen Prinzips, München 1972, S. 9 ff.; Bodo Dennewitz, Der Föderalismus, sein Wesen und seine Geschichte, Hamburg 1947, S. 15 ff..

110

So auch - jedoch ohne direkten Bezug zum Sudan - Allemante G. Selassie, Ethnic Federalism: Its Promise and Pitfalls for Africa, Yale J. Int'1 L. 30/1, New Haven (Winter) 2003, S. 51 (85). 
aufgeführt, dass ,die föderale Regierung eine der Forderungen des Volkes seit 1955 gewesen sei“, die „föderale Regelung die Lösung aller Probleme darstelle“ und Föderalismus für den heterogenen Sudan „the road to unity in diversity“ sei. Auch wenn letzterem grundsätzlich zugestimmt werden kann, ist dem Sudan trotz entsprechender Selbstbezeichnung ein föderativer Charakter bislang abzusprechen, weil die Zentralgewalt über so viel Macht verfügt, dass sie jederzeit de facto unbeschränkt auf Exekutive und Legislative der Regionen qualitativ und quantitativ Einfluss nehmen kann. ${ }^{111}$

Dieser status quo macht deutlich, dass zum Schutz, aber auch zur Sicherung der verfassungsrechtlich anvisierten föderalen Struktur eine stark ausgebaute und effektive Verfassungsgerichtsbarkeit ${ }^{112}$ dringend erforderlich ist. Wie es Brun-Otto Bryde bereits zutreffend formuliert hat, braucht man die deutsche Neigung, Verfassungsrecht nur dann ernst zu nehmen, wenn es justitiabel ist, nicht zu teilen, um zu erkennen, dass die Justitiabilität dem Verfassungsrecht eine grundlegend andere Bedeutung gibt. ${ }^{113}$ Dies trifft umso mehr auf die sudanische Verfassungsentwicklung zu, ist man sich doch weitgehend darüber einig, dass man nicht wieder in alte Muster zurückfallen will.

\section{Zum Selbstbestimmungsrecht}

Das Selbstbestimmungsrecht der Völker bildet einen materiellen Grundpfeiler der modernen Völkerrechtsordnung. Es findet sich ausdrücklich erwähnt in Art. 1 Nr. 2, 55 UNCharta und in der Dekolonialisierungsdeklaration von 1960, in der Friendly RelationsDeklaration von 1970, der Aggressionsdefinition von 1974 sowie in Art. 20 (1) der African [Banjul] Charter on Human and People's Rights von 1981. Impliziert liegt es ferner den Regelungen der Kapitel XI - XIII der Charta der Vereinten Nationen zugrunde.

Ausführlicher Hanspeter Mattes, Die Umgestaltung des Sudan in einen "föderalen“ Bundesstaat 1991, in: Nord-Süd aktuell, 11/1, Hamburg 1997, S. 102 (112), Rainer Tetzlaff, Ethnische Konflikte im Sudan, a.a.O. (Fn. 2), S. 152; jeweils mit weiteren Verweisen. Sowie Ahmed T. el-Gaili, Federalism and the Tyranny of Religious Majorities: Challenges to Islamic Federalism in Sudan, Harvard Int'l L. J. 45/2, Cambridge (Summer) 2004, S. 503 (544); Angelo L. Loiria, The Search for a Permanent Constitution in Sudan: Political Implications, in: Hassan M. Salih, et. al. [Hrsg.], Federalism in the Sudan, Khartum 1995, S. 108.

112

Unter einem zukunftsweisenden Aspekt sehr anschaulich zum Supreme Constitutional Court Ägyptens (al-mahkkama ad-dustūriya al-`ulyā), dessen Rolle, Funktion und Auslegung eines solchen Verfassungsartikels: Baber Johansen, a.a.O. (Fn. 104), S. 881 - 896; Kilian Bälz, a.a.O. (Fn. 104), S. 178 - 179.

113

Brun-Otto Bryde, Überseeische Verfassungsvergleichung nach 30 Jahren, VRÜ (30), Hamburg 1997, S. 452 (461). 
Hinsichtlich der Wirkungsrichtung kann das Selbstbestimmungsrecht in eine innere und eine äußere Komponente aufgeteilt werden. Bezüglich der Art der Ausübung unterscheidet man innerhalb dieser Komponenten zwischen offensivem und defensivem Selbstbestimmungsrecht. ${ }^{114}$ Zum inneren Selbstbestimmungsrecht gehört demnach, dass ein Volk, das nach Bildung eines Staates zu einem Staatsvolk wurde, frei über seine Regierungsform bestimmen kann. Dazu gehört aber auch, dass ethnische Minderheiten ihre Eigenarten bewahren und verteidigen können. Diesem defensiv inneren Charakter steht die offensiv innere Ausprägung der Selbstbestimmung unmittelbar gegenüber. Typisch dafür wäre beispielsweise die Sezession einer Minderheitengruppe aus dem bestehenden Staatenverbund. 115

Die nicht nur im Sudan verbreitete Aussage „unity in diversity “116, spiegelt in diesem Zusammenhang das staatsrechtliche Ringen in pluralistischen Gesellschaften um Methoden und Mechanismen wider, die das (defensiv innere) Selbstbestimmungsrecht bestimmter Volksgruppen einerseits bewahren soll und dem adäquaten Bedürfnis nationaler Einheit andererseits. Um hier die optimale Balance zwischen den beiden konträren Polen der Sezession als (offensiv inneres) Selbstbestimmungsrecht und der territorialen Integrität zu finden, bemüht man sich insbesondere in den Staaten am Horn von Afrika - Vielvölkerstaaten par excellence - seit geraumer Zeit und mit zunehmender Tendenz, dieser Situation mit praktikablen verfassungsrechtlichen Ansätzen zu begegnen.

\section{a. Defensiv innere Selbstbestimmung}

Die jahrzehntelangen sudanischen Bemühungen um praktikable verfassungsrechtliche Lösungen sind als theoretischer Ansatz im Sinne eines progressiv defensiven inneren Selbstbestimmungsprozesses bemerkenswert, wenngleich er in der Verlaufsgeschichte immer wieder erhebliche Rückschläge hinnehmen musste.

Felix Ermacora, Autonomie als innere Selbstbestimmung, ArchVR 38, Tübingen 2000, S. 285 ff.; Dietrich Murswiek, Offensives und defensives Selbstbestimmungsrecht, in: Der Staat, 23, Berlin 1984, S. 523.

Vollständigkeitshalber sei hier noch das äußere Selbstbestimmungsrecht erwähnt: Darunter versteht man im Allgemeinen das Recht eines souveränen Staates, sich einem anderen Staat anzuschließen. Ebenso steht auch ethnischen Minderheiten ein solches Recht zu, soweit man ihnen ein Sezessionsrecht zugesteht. Weiterführend kann an dieser Stelle auf die Thematik nicht eingegangen werden. Vgl. dazu Kristin Henrard / Stefaan Smis, Recent Experiences in South Africa and Ethiopia to Accommodate Cultural Diversity: A Regained Interest in the Right of Self-Determination, in: Journal of African Law 44, London 2000, S. 17 (22 ff.) mit zahlreichen weiteren Verweisen.

116

Dieser, nicht erst in den Verhandlungen aufgekommene Gedanke, wurde im Power Sharing Agreement schriftlich fixiert. Vgl. beispielsweise Part I (General Principles), 1.4.2. 
So gewährte das unter Vermittlung des äthiopischen Kaiser Ḩāyla Śellāsē schon 1972 in Addis Abeba unterzeichnete und 1973 in die Verfassung inkorporierte Abkommen den drei genannten Südprovinzen Autonomie. Es konzedierte das in der Verfassung verbriefte Recht, ein eigenes Parlament, die Regionalversammlung in Juba, und eine eigene Regierung, den High Executive Council, wählen zu können. Der Süden sollte von einem Regionalpräsidenten regiert werden, der von dem nationalen Präsidenten auf Vorschlag der Regionalversammlung ernannt wurde. ${ }^{117}$ Doch in der Praxis verhinderte der Anspruch der Militärdiktatur Numairis auf Alleinherrschaft die Herausbildung eines wirklich kooperativen Föderalismus. Dem Süden wurde das Recht auf eigene Steuererhebung und Finanzplanung vorenthalten. Kompetenzen wie die Außenpolitik, die Verteidigung, Währung, Wirtschaft und Sozialplanung verblieben gemäß Chapter IV, Art. 7 des Abkommens bei der Zentralregierung. Trotz des theoretisch guten Ansatzes, bestanden auch im 11-jährigen Zeitfenster von 1972-1983 keine echten Föderalismusmaßnahmen, die volle innerstaatliche Umsetzung erfolgte letztlich nicht. Darüber hinaus, heute allerdings weitgehend vergessen, war auch die Autonomieverwaltung von Streitigkeiten, Ineffizienz und Korruption geprägt. ${ }^{118}$

Selbst Friedensgespräche, die im Mai 1992 in der nigerianischen Hauptstadt abgehalten wurden, führten zu keinen Fortschritten. Im Schlusskommuniqué wurde eine mögliche Selbstbestimmung des Südens nicht einmal erwähnt.

\section{b. Offensiv innere Selbstbestimmung}

In der dritten Verfassungskodifikation Äthiopiens seit 1931, der äthiopischen Verfassung von 1994, zeigen sich beispielsweise eine Fülle neuer Ansatzpunkte, wenn man sie mit anderen verfassungsrechtlichen Versuchen in Afrika und speziell im Sudan vergleicht. Aufbauend auf Art. 2 der Transitional Period Charter ${ }^{119}$, dem verfassungsrechtlichen

Addis Ababa Agreement on the Problem of Southern Sudan, Chapter VI (The Executive), Art. 19.

118 Vgl. Rainer Tetzlaff, Bürgerkrieg und Staatsbildung im Sudan: Ein Land auf der Suche nach seiner kulturellen Identität, a.a.O. (Fn. 2), S. 391 f.; Edgar O'Ballance, Sudan. Civil War and Terrorism. 1956 - 99, London / New York 2000, S. 206; Rüdiger Wolfrum, a.a.O. (Fn. 33), S. 1250; Hanspeter Mattes, Die Umgestaltung des Sudan in einen "föderalen“ Bundesstaat 1991, a.a.O. (Fn. 111), S. 103; Siehe auch Peter Nyot Kok, Governance and Conflict in the Sudan, 1985-1995, Hamburg 1996, S. 72-81.

119 Art. 2 der Transitional Period Charter Äthiopiens vom 22. Juli 1991

The right of nations, nationalities and peoples to self-determination is affirmed. To this end, each nation, nationality and people is guaranteed the right to:

a) Preserve its identity and have it respected, promote its culture and history and use and develop its language;

b) Administer its own affairs within its own defined territory and effectively participate in the central government on the basis of freedom, and fair and proper representation; 
Übergangsregime Äthiopiens von 1991 bis 1994, garantiert die äthiopische Verfassung in Art. $39^{120}$ als letzte Konsequenz eine (offensiv innere) Selbstbestimmung in Form des ius secessionis $^{121}$. $^{122}$ Nicht einmal Südafrika und Namibia ${ }^{123}$ haben in ihren Konzeptionen den Dezentralisierungsgedanken soweit vorangetrieben. ${ }^{124}$

Bei der allzu verständlichen Euphorie im Südsudan bezüglich einer möglichen Sezession nach der sechsjährigen Übergangsperiode, sollte man dennoch nicht die bisherigen Errungenschaften vorschnell als Vorbereitungen zur Trennung in zwei souveräne staatliche

c) Exercise its rights to self-determination of independence, when the concerned, nation/nationalities and people is convinced that the above rights are denied, abridged or abro120 gated.

Art. 39 der äthiopischen Verfassung:

(1) Every Nation, Nationality and People in Ethiopia has an unconditional right to self-determination, including the right to secession.

(2) Every Nation, Nationality and People in Ethiopia has the right to speak, to write and to develop its own language; to express, to develop and to promote its culture; and to preserve its history.

(3) Every Nation, Nationality and People in Ethiopia has the right to a full measure of selfgovernment which includes the right to establish institutions of government in the territory that it inhabits and to equitable representation in state and Federal governments.

(4) The right to self-determination, including secession, of every Nation, Nationality and People shall come into effect:

(a) When a demand for secession has been approved by a two-thirds majority of the members of the Legislative Council of the Nation, Nationality or People concerned;

(b) When the Federal Government has organized a referendum which must take place within three years from the time it received the concerned council's decision for secession;

(c) When the demand for secession is supported by majority vote in the referendum;

(d) When the Federal Government will have transferred its powers to the council of the Nation, Nationality or People who has voted to secede; and

(e) When the division of assets is effected in a manner prescribed by law.

(5) A "Nation, Nationality or People" for the purpose of this Constitution, is a group of people who have or share large measure of a common culture or similar customs, mutual intelligibility of language, belief in a common or related identities, a common psychological make-up, and who inhabit an identifiable, predominantly contiguous territory.

Vgl. Felix Ermacora, a.a.O. (Fn. 114), S. 287.

122 An dieser Stelle sei noch klargestellt, dass Eritrea durch ein Referendum unter VN-Aufsicht am 24.5.1993, der Anerkennung durch Äthiopien und am 28.5.1993 durch den Beitritt Eritreas zu den Vereinten Nationen, nicht von dieser verfassungsrechtlichen Regelung einer Sezession Gebrauch machte.

123

Namibia erfährt seit seiner Unabhängigkeit 1990 einen tiefen innerterritorialen Wandel und steht ebenso vor der Herausforderung des Aufbaus autonomer Gebietskörperschaften an den Peripherien. Herrschaftsordnung Äthiopiens, VRÜ (1993), Hamburg 1993, S. 166 ff.; ders., Die neue äthiopische Verfassung als konkurrierende Grundlage der Herrschaftsordnung Äthiopiens, VRÜ (30), Hamburg 1997, S. 181 ff. 
Einheiten qualifizieren. Der Interimsperiode muss in jeder Hinsicht die Bedeutung zuteil werden, welche ihr zugedacht ist, nämlich vorerst unter der Prämisse einer defensiv inneren Selbstbestimmung in gemeinsamer Kraftanstrengung auf eine befriedete staatliche Einheit hinzuwirken. ${ }^{125}$ Gerade weil die „Gräben“ zwischen den Konfliktparteien so tief sind, ist es ungewiss, welche Folgen sich aus einer Teilung ergeben könnten. Die Zukunft des Sudans hängt auch untrennbar mit dem fragilen Frieden am Horn von Afrika zusammen, zumal nicht nur die genaue territoriale Trennung eines südlichen Teilstaates nach wie vor umstritten ist. Die Bedenken der angrenzenden Staaten sind in jedem Falle ernst zu nehmen. Insbesondere die mehrfach geäußerten Befürchtung eines islamistischeren Charakters in einem fragmentierten Reststaat im Norden und des damit möglicherweise verbundenen sicherheitspolitischen Risikos sind ebenso ernst zunehmen wie die Besorgnis der ohnehin im Zusammenhang mit den völkerrechtlichen Verträgen von 1929 und 1959 strittigen und hochbrisanten Neuverteilung des Nilwassers. ${ }^{126}$

Dieses axiomatische Leitmotiv kommt durch die Formulierung im Power Sharing Agreement deutlich zum Ausdruck, welcher wiederum Ausfluss des Grundgedankens von Machakos ist.

Machakos-Protocol, Part A (Agreed Principles), 1.1:

That the unity of the Sudan, based on the free will of its people democratic governance, accountability, equality, respect, and justice for all citizens of the Sudan is and shall be the priority of the parties and it is possible to redress the grievances of the people of South Sudan and to meet their aspirations within such a framework.

Power Sharing Agreement, Part I (General Principles):

1.4 The Parties agree to that the following principles shall guide the distribution of powers and the establishment of structures:

1.4.1 Recognition of both the sovereignty of the nation as vested in its people as well as the need for autonomy of the Government of Southern Sudan and States throughout the Sudan;

1.4.2 Affirmation of the need for both national as well as state and Southern norms and standards so as reflect the unity of the country and the diversity of the Sudanese people;

1.4.4 Recognition of the need for the involvement and participation of the people of South Sudan at all levels of government and National institutions as an expression of the $[\ldots]$ national unity of the country;

1.4.5 Pursuit of good governance, accountability, transparency, democracy, and the rule of law at all levels of government to achieve lasting peace; $[\ldots]$.

Weiterführend zur Problematik: a) Allgemein; Christian Ule, Das Recht am Wasser: dargestellt am Beispiel des Nils, Baden-Baden 1998; Omaia Elwan, Nile River, in: Rudolf Bernhardt [Hrsg.], The Encyclopedia of Public International Law (EPIL), 3, Amsterdam [u.a.] 1997, S. 589 595; Ashok Swain, The Nile River Basin Initiative, in: SAIS-Review 22/2, Washington D.C. (Summer-Fall) 2002, S. 293-308; b) im Falle einer Sezession; Michael A. Lange, Strategische Bedrohung aus dem Süden - Umverteilung des Nilwassers?, in: Kairo-News Nr. 3, Konrad Adenauer Stiftung, Kairo 2004, S. 5 -7; ders., Ägypten und die Friedensbemühungen im Sudan, in: KAS-AI, (9/02), S. 32 (35 ff.) und c) beim Fortbestand der staatlichen Einheit, der Weiterführung des 1978-1984 begonnenen, umstrittenen Jonglei-Kanal-Projekts und dessen potentiellen politischen Sprengstoff; Peter Giovannini, Der Sudan zwischen Krieg und Frieden, Wien 1988, S. 126129; Manuel Schiffler, Konflikt am Nil, in: INAMO Nr. 5/6, Erlangen 1987, S. 9-14. 
Vieles wird auch von der wirtschaftlichen Entwicklung abhängen, vor allem vom Gelingen des Wiederaufbaus im Süden. Nachdem man sich darauf einigen konnte, dass die Zentralregierung (zusammen mit den nördlichen Gliedstaaten) und die südsudanische Regierung jeweils $50 \%$ von den Einnahmen aus dem steigenden Ölförderungen erhalten, besteht diesbezüglich berechtigte Hoffnung. ${ }^{127}$ Der essentielle Grundpfeiler ist damit gesetzt worden.

Die kurz bevorstehende Einführung der dezentralisierten Systemstruktur kann davon losgelöst als pragmatische politische Entscheidung gesehen werden. Hierbei ist nicht allein die „Kriegsmüdigkeit“ Grund für den verfassungsrechtlichen Kurswechsel gewesen. ${ }^{128}$ Vielmehr scheint es so, als hätten die Machthaber erkannt, dass die Überwindung des innerstaatlichen Nord-Süd Konflikts nur auf der Basis einer tatsächlichen föderalistischen Struktur möglich ist. Die zentrale Kontrolle des Gesamtstaates, gespeist von ihrer bisherigen Reflexion einer gesamtgesellschaftlichen, islamistischen Vision, vermag offenbar den aufkommenden ökonomischen Bedürfnissen des Staates nicht mehr gerecht zu werden. ${ }^{129}$

\section{Zur Relevanz der šari`a}

In der bis zum Sturz Numairis im April 1985 in Kraft gebliebenen „Ständigen Verfassung des Sudans“ („Sudan Permanent Constitution“) finden sich weitere Ergebnisse des Addis Ababa Agreement on the Problem of Southern Sudan. Obwohl während der Debatten der verfassunggebenden Versammlung von 1972 bis 1973 einige Mitglieder gefordert hatten, so wie im Verfassungsprojekt von 1968 vorgeschlagen, einzig den Islam als Staatsreligion anzuerkennen, scheiterte dies am Widerstand der Mehrheit der Versammlung. Durch Art. 16 (c) der Verfassung wurden das Christentum und andere religiöse Überzeugungen offiziell anerkannt. ${ }^{130}$

Ohne größeren Widerstand angenommen wurde dagegen Art. 9, der das islamische Recht und das Gewohnheitsrecht als Hauptquellen der Gesetzgebung bestimmte. Wurde diese formelle Festschreibung weitgehend, wenn auch umstritten, als gleichberechtigte Rechts-

127 Wealth Sharing Agreement 5.6. Darüber hinaus hat man sich in Ziffer 5.5 des Wealth Sharing Agreements darauf verständigt, dass mindestens $2 \%$ der Öleinnahmen den Ölproduzierenden Staaten/Regionen im Verhältnis zur Förderungsleistung zugewiesen wird.

Yusuf Fadl Hasan, a.a.O. (Fn. 3), S. 23

129 Ähnlich auch schon im Jahre 1997; Hanspeter Mattes, Die Umgestaltung des Sudan in einen 130 "föderalen" Bundesstaat 1991, a.a.O. (Fn. 111), S. 112.

Art. 16 Sudan Permanent Constitution:

(c) Heavenly religions and the noble aspects of spiritual beliefs shall not be insulated or held in contempt. 
quellen verstanden, so diente Numairi dieser Artikel im Jahre 1983 als verfassungsrechtliche Interpretationsgrundlage zur Einführung der $\check{\text { šari }}{ }^{`} a$ als maßgebliche Grundlage der Rechtsordnung. Er erließ im September 1983 die der sudanischen Volksversammlung vorgelegten, aber von dieser bis dahin nicht verabschiedeten Gesetzesentwürfe, als provisorische Präsidialbeschlüsse. Grundlage war die Ermächtigung in der Verfassung, beim Vorliegen von eiligen und unaufschiebbaren Umständen Gesetze in Form von provisorischen Beschlüssen zu erlassen. ${ }^{131}$ Die Einführung des islamischen Strafgesetzes führte dann, nach Auflösung der Regionalversammlung und Neugliederung der südlichen Provinzen, in letzter Konsequenz mit zum Bruch des Addis Ababa Agreement on the Problem of Southern Sudan. Das entfachte die kriegerische Auseinandersetzung von neuem.

Die Betrachtung der politischen Vorgeschichte der Einführung der Septembergesetze macht deutlich, dass der Zeitpunkt auch damals zum großen Teil durch die politische und wirtschaftliche Notlage, in die das Regime bis 1983 geriet, bedingt war. ${ }^{132}$ Seither bemüht sich die SPLM unter der Führung Garangs um das Konzept eines säkularen Staates, der die Religionsfreiheit aller Bürger respektiert und ethnisch-regionale Diskriminierungen beseitigt. $^{.133}$

Die gegenseitigen Zugeständnisse im Machakos-Protokoll, wonach $\check{s} \operatorname{ari}^{`} a$ als Quelle der nationalen Gesetzgebung nur für Gliedstaaten außerhalb des Südsudans gelten soll ${ }^{134}$, sind im historischen Kontext natürlich wieder ein enormer Fortschritt, zudem sich die šari $a$ in den vergangenen zwei Dekaden - als Synonym für einen nicht-säkularen Staat - zu einem der Haupthindernisse entwickelt hat. ${ }^{135}$ Das bedeutet aber nicht, dass im Verhältnis von Staat zu Religion eine umfassende Einigung erzielt wurde. Sieht man von Personalstatutsfragen ab, zu denen das Familien- und Erbrecht zählen und die jeweils dem religiösen

132 Olaf Köndgen, Das islamische Strafrecht des Sudans. Von der Einführung 1983 bis zum Juli 1992, Hamburg 1992, S. 27 ff; Omaia Elwan, Gesetzgebung und Rechtsprechung, a.a.O. (Fn. 103), S. 221 (243); Hizkias Assefa, Mediation of Civil Wars: Approaches and Strategies - The Sudan Conflict, London / Boulder 1987, S. 211; Peter Nyot Kok, Codifying Islamic Absolutism in the Sudan: A Study in Constitution-Making under al-Beshir, ORIENT 36 (4/1995), Hamburg 1995, S. 673 ff.; Reinhard Fischer, Sudan im Überblick, INAMO Nr. 11, Erlangen (Herbst) 1997, S. 4 (6); Douglas H. Johnson, Political and Economic Consequences Beyond Assistance, in: Geoff Loane / Tanja Schümer [Hrsg.], The Wider Impact of Humanitarian Assistance - The Case of Sudan and the Implication for European Union Policy, Baden-Baden 2000, S. 45 (50).

Rüdiger Seesemann, Der Sudan und die islamische Verfassung, in: INAMO 17, Berlin 1999, S. 42; Olaf Köndgen, a.a.O. (Fn. 132), S. 21 und 81; Peter Mattes, Sudan, a.a.O. (Fn. 2), S. 253; Mansour Khalid, a.a.O. (Fn. 3); Ulrich Delius, Der Konflikt im Sudan - keine Hoffnung auf Frieden, a.a.O. (Fn. 9), S. 191; Peter Nyot Kok, Die Jihâd-Konzeption der sudanischen Armee, in: Hanspeter Mattes / Sigrid Faath [Hrsg.], Wuquf 7-8, Hamburg 1993, S. 167 (168-169). 
Recht beziehungsweise dem Gewohnheitsrecht unterliegen, so werden sich wohl Schwierigkeiten in der Anwendung der übrigen islamrechtlich geprägten Bereiche, insbesondere im Strafrecht ergeben. De facto wurden die strafrechtlichen Normen der šari ${ }^{\complement} a$ im Süden zwar praktisch nahezu nie angewendet, wohl aber auf die im Norden lebenden Südsudaner. ${ }^{136}$ Unter dem Gesichtspunkt einer Vereinbarkeit mit Grundrechten zeichnen sich hier zahlreiche Fragen ab, die es künftig zu beantworten gilt. ${ }^{137}$

Sicherlich, diese Rechte sollen expressis verbis in Khartum gewährleistet und eingehalten werden $^{138}$, und nach Maßgabe von Ziffer 2.4.5.4 des Power Sharing Agreement sollen sich die Richter bei ihrer Entscheidung in Fällen, bei denen in der Hauptstadt ${ }^{139}$ lebenden Nichtmuslime involviert sind, in ihrem Ermessen unter anderem von dem klassischen shariatsrechtlichen Prinzip leiten lassen, dass Nichtmuslime bei der Verhängung von Strafen nicht den islamrechtlichen Regelungen unterlie

gen. Dazu soll sogar ein spezieller generalstaatsanwaltlicher Bezirk zur umfassenden Überwachung geschaffen werden. ${ }^{140}$

Damit zusammenhängend ist jedoch der rechtliche Status der Hauptstadt noch nicht genau geklärt. Ursprünglich wurde ein Gliedstaat sui generis, etwa vergleichbar mit Washington D.C., angedacht, in welcher das Shariatsrecht nicht maßgeblich sein soll. Da aber die SPLM die šari ${ }^{\complement} a$ neben dem ,consensus of the people “ als Gesetzgebungsquelle außerhalb der Gebiete des Südsudans anerkannt hat, streng genommen also eigentlich auch die im Norden liegende Hauptstadt Khartum, bleibt abzuwarten, wie sich ihr Status - unter dem Blickwinkel einer partizipatorischen Verwaltung ${ }^{141}$ - weiterentwickelt. Ob in diesem Kontext überhaupt die beabsichtigte Kommission zur Sicherung der Rechte von Nichtmuslimen von einer Präsidentschaft, die sich aus zwei Persönlichkeiten des gegenwärtigen nordsudanischen Regimes und vergleichsweise einzigen südsudanischen Repräsentanten zusammensetzt, einberufen wird, ist fraglich. Ihre Aufgabe, negative Auswirkungen auf Rechte von

So Olaf Köndgen, a.a.O. (Fn. 132), S. 48. Andere Auffassung Jürgen Adam, Die Lage der Menschenrechte im Sudan 1985 -1993, in: Hanspeter Mattes / Sigrid Faath [Hrsg.], Wuquf 7-8, Hamburg 1993, S. 101 (113); allerdings ohne einen Beleg oder nähere Ausführungen dafür zu liefern, dass die von ihm als „Tatsache“ dargestellte Anwendung der menschenverachtenden Handlungen in den Kriegswirren tatsächlich auch auf die Septembergesetze zurückzuführen sind und sich eben nicht als faktischen Gegenstand der Kriegsführung darstellte.

Abullahi A. An-Na'im / Francis M. Deng, Self Determination and Unity: The Case of Sudan, in: Keith Hawkins [Hrsg.], Law and Policy, 18, No. 3 \& 4 (July / Oct. 1996), S. 199 (217); Rüdiger Wolfrum, a.a.O., (Fn. 33), S. 1261.

139

Power Sharing Agreement, Part II (Institutions at the National Level), 2.4.3.

Gemäß Power Sharing Agreement, Part II (Institutions at the National Level), 2.4.1 wird dies auch weiterhin Khartum sein.

140 Power Sharing Agreement, Part II (Institutions at the National Level), 2.4.7.3.

${ }^{141}$ Vgl. Power Sharing Agreement, Part II (Institutions at the National Level), 2.4.2. 
Nichtmuslimen durch die Anwendung der šari $a$ in der Hauptstadt zu überwachen, hätte ohnehin nur empfehlenden und nicht konstituierenden Charakter. ${ }^{142}$ Bei einer Anzahl von zwischenzeitlich über 2 Millionen Nichtmuslimen und Binnenflüchtlingen, die sich um die nördlichen Städte, insbesondere in (und um) die Hauptstadt angesiedelt haben, ${ }^{143}$ drängt sich dann natürlich unwillkürlich - wenn auch nicht nur - die Frage der Fortgeltung der bereits angegebenen islamrechtlich-geprägten Regelungen auf. Die künftige staatsrechtliche Konzeption und das Verhältnis von Staat zu Religion sind also keineswegs so sicher geklärt, wie es auf den ersten Blick den Anschein erwecken mag.

Das ist auch nicht verwunderlich, wenn man bedenkt, dass dieser verfassungsrechtliche Diskurs schon unmittelbar nach der Unabhängigkeit begann und die politischen Spannungen seither prägt. ${ }^{144}$ Die historische Darlegung macht deutlich, dass diese neuralgischen Punkte unbedingt weiterführender Auseinandersetzung bedürfen. Der Disput zwischen Aḥmad al-Bašīr und Ḥasan at-Turābī ist dabei nur ein sehr schmaler Ausschnitt im politisch-ideologischen Spektrum des Sudan.

Viele der Programme zur Förderung der Zivilgesellschaft bedürfen zudem einer kritischen Evaluation. Hier wird oft mit Vertretern zusammen gearbeitet, die die intellektuelle, säkulare, oft im „Westen“ ausgebildete Elite stellen und sich auf dem internationalen Parkett bewegen. In ihrer eigenen Gesellschaft sind sie aber oft nur noch wenig verwurzelt. Damit mangelt es ihnen an der Basis, um effektive politische Bewegungen zu bilden, die Reformen wirkungsvoll einfordern, Interessen der Bevölkerung bündeln und in den politischen Willensbildungsprozess einbinden können. ${ }^{145}$ Gerade im ,islamischen Orient“ hat sich gezeigt, dass Modernisierung und Demokratisierung von oben nicht funktionieren. Daran scheiterten die Pahlavys im Iran, und die Türkei hat bis heute erhebliche Schwierigkeiten, mit den Folgen einer derartigen Modernisierung fertig zu werden. Das für den Irak konzipierte Modell kann nicht einmal im Irak durchführbar sein, vom Paradebeispiel für den gesamten islamisch-geprägten Nahen Osten ganz zu schweigen.

${ }^{142}$ Vgl. Power Sharing Agreement, Part II (Institutions at the National Level), 2.4.6.

143 Marina Peter, Vertriebene im eigenen Land - "displaced persons" aus dem Südsudan im Großraum Khartum, in: Hanspeter Mattes / Sigrid Faath [Hrsg.], Wuquf 7-8, Hamburg 1993, S. 547 (549 f.); Richard Gray, Epilogue, in: Yusuf Fadl Hasan / Richard Gray [Hrsg.], Religion and Conflict in Sudan, Nairobi 2002, S. 195 (198); Melha Rout Biel, Geschichte des sudanischen Konflikts, in: Melha Rout Biel [Hrsg.], Der Sudan zwischen Krieg und Frieden, Marburg 2003, S. 30; Ahmed T. el-Gaili, a.a.O. (Fn. 111), S. 503 (543); Reinhard Fischer, a.a.O. (Fn. 132), S. 6; Rüdiger Seesemann, a.a.O. (Fn. 133), S. 43.

145 Abullahi A. An-Na'im / Francis M. Deng, a.a.O. (Fn. 137), S. 213.

Muriel Asseburg, Demokratisierung im Nahen und Mittleren Osten, in: Flüsse verbinden (Publikation der Heinrich-Böll-Stiftung), S. 8. 
Umso wichtiger ist es im Sudan, den vielschichtigen geistig-politischen Charakter nicht auf die Protagonisten zu reduzieren. In Zeiten, die durch Schlagworte wie „Friedenssicherung“ und ,internationale Sicherheit“ geprägt sind, ist eine Bündelung aller inneren und äußeren Kräfte dringend erforderlich. Die staatliche Monopolisierung und damit verbundene allgemeine Wahrnehmung des Islam täuscht darüber hinweg, dass die Bestimmungen der $\check{s}^{\prime} r^{\complement} a$ keineswegs a priori die Durchsetzung der Menschen- und Grundrechte verhindert. Das Engagement und die Bemühungen moslemischer Reformtheologen werden nach wie vor nicht angemessen berücksichtigt. ${ }^{146}$ Missverständnisse, Misstrauen und nicht zuletzt hinderliche Sprachbarrieren führen zu einer Verzerrung und scheinen einen konstruktiven Dialog zu hemmen.

\section{Zum weiteren Friedensprozess}

Wie die IGAD-Friedensbemühungen gezeigt haben, sind hier regionale Organisationen mit den politisch-ideologischen Gegebenheiten oftmals besser vertraut und nachweislich, aufgrund ihrer regionalen Verankerung, hervorragend dazu geeignet, offiziell-schlichtend einzuschreiten. ${ }^{147}$ Dass die IGAD-Verhandlungen bisher jedoch nur zwischen der SPLM und der Zentralregierung stattfanden, steht einem dauerhaften und umfassenden Frieden im Sudan und damit der Stabilität des Landes im Wege. Dies verdeutlichen nicht nur die entsetzlichen Geschehnisse in Dārfūr. ${ }^{148}$ Auch die weitere Entwicklung in der im Osten des Sudan gelegenen Beja- (Biğā-)Region sollte aufmerksam beobachtet werden. Obwohl derzeit relativ ruhig, ist ein Wiederaufflackern bewaffneter Auseinandersetzungen auch dort nicht auszuschließen. Insbesondere Port Sudan am Roten Meer hat strategische Bedeutung, denn es ist der einzige Hafen, über den das Land Erdöl ausführt und Waren importiert. All dies verdeutlicht umso mehr, dass ohne eine politische Lösung der verschiedenen Konflikte im Sudan, auch nach den formellen Zugeständnissen gegenüber der SPLM, eine Befriedung des Landes nicht denkbar ist.

146

Omaia Elwan, Rechtsnormen im Islam und Menschenrechte, in: Islam eine andere Welt?, Heidelberg 2002 (2. Auflage), S. 73 f.; Hans-Georg Ebert, Arabische Verfassungen und das Problem der „,islamischen“ Menschenrechte, VRÜ (30), Hamburg 1997, S. 520 (531); Heiner Bielefeldt, Muslime zwischen Sharia und Menschenrechten, Internationales Afrikaforum 30/1, Köln 1994, S. 65 (69).

147 Mansour Khalid, a.a.O. (Fn. 3), S. 370 f.; vgl. dazu auch Ulrich K. Preuß / Peter Tobias Stoll / Joscha Schmierer / Birgit Laubach, Die Rolle des Völkerrechts in einer globalisierten Welt, Berlin 2004, S. 14.

Dazu unter anderem Olaf Köndgen, Tragödie in Darfur, KAS-AI (10/04), Seite 4 - 1; Hanspeter Mattes, Sudan Sommer 2004: Frieden im Süden, Krieg im Westen, Konflikt mit der Opposition, a.a.O. (Fn. 2), S. 288 - 290; Khalid Y. Khalafalla, Der Konflikt in Darfur (Übersetzung aus dem Englischen durch Martina Boden), in: APuZ 4/2005, Bonn 2005, S. 40 - 46. 
Nordsudanische Oppositionsparteien, bedeutsame zivilgesellschaftliche Gruppen, die der Militärdiktatur und/oder der Praxis der islamischen Gesetzgebung ablehnend gegenüberstehen, wurden in die Verhandlungen nicht eingebunden. Außerdem hat sich die sudanische Regierung in 1.6.1 (Satz 1) des Power Sharing Agreements ausdrücklich dazu bekannt "to comply fully - throughout the country - with its obligations under the international human rights treaties to which it is or becomes a party”. Es ist zu hoffen, dass dieses rühmliche Vorhaben gerade in der bevorstehenden schwierigen Phase künftig landesweit umgesetzt wird.

Auch die SPLM kann wegen der Nubaberge in erhebliche Bedrängnis kommen. Hat sich dort doch die Bevölkerung dafür ausgesprochen, dass die SPLM sie in den Verhandlungen vertritt. Sollten sich ihre Befürchtungen bestätigen, dass ihre Interessen nicht ausreichend berücksichtigt werden, könnten auch in dieser Region erneut kriegerische Auseinandersetzungen aufflammen. So muss auch die SPLM erhebliche Kraftanstrengungen leisten, um zivilgesellschaftliche Gruppen und andere politische Strömungen im Süden einzubinden. ${ }^{149}$ Deren Kritik, die SPLM/A habe sich für die sechsjährige Übergangsperiode ein Machtmonopol gesichert und alle anderen politische Kräfte von der politischen Beteiligung ausgeschlossen, muss ernst genommen werden. Eine solche Situation würde dem Aufbau demokratischer Strukturen im Süden die Grundlage entziehen. Selbst SPLM/A Führer Garang muss sich wegen seines autoritären Führungsstils künftig mehr mit der Kritik in den eigenen Reihen auseinandersetzen, nachdem er es in der Vergangenheit vorgezogen hatte, politische Rivalen mehr oder minder gewaltsam auszuschalten. ${ }^{150}$

149

Zu den wechselnden inner-südsudanischen Strömungen - selbst innerhalb der SPLM/A - siehe Melha Rout Biel, a.a.O. (Fn. 143), S. 26 f. ('4.4 Sind die Führer der Befreiungsbewegung im Südsudan wirklich Befreier?'); Ankush B. Sawant, Ethnic Conflict in Sudan in Historical Perspective, in: International Studies 35/3, New Dehli (July-September) 1998, S. 343 (359); Mansour Khalid, a.a.O. (Fn. 3), S. 332 - 336 ('Cleavages in the South: A Conflict within the Conflict'); Ulrich Delius, Der Konflikt im Südsudan - keine Hoffnung auf Frieden, a.a.O. (Fn. 9) S. 194 f.; Hanspeter Mattes, Sudan, a.a.O. (Fn. 2), S. 253; Scott Peterson, Me against my Brother - At War in Somalia, Sudan and Rwanda, New York / London 2000, S. 219. Zur Thematik „Dinka Vorherrschaft" in den südlichen Landesteilen vgl. Peter Nyot Kok, a.a.O. (Fn. 118), S. 73; Ankush B. Sawant, Ibid., S. 359; Douglas H. Johnson, a.a.O. (Fn. 4), S. 51 ff; Scott Peterson, Ibid, S. 203 und 219.

150

Rainer Tetzlaff, Bürgerkrieg und Staatsbildung im Sudan: Ein Land auf der Suche nach seiner kulturellen Identität, a.a.O. (Fn. 2), S. 395 ff.; Vgl. auch zur historischen SPLA-Spaltung Sharon Elaine Hutchinson, Spiritual Conflicts of an unfinished War, in: Yusuf Fadl Hasan / Richard Gray [Hrsg.], Religion and Conflict in Sudan, Nairobi 2002, S. 137; Yehudit Ronen, Sudan, in: Bruce Maddy-Weitzman [Hrsg.], Middle East Contemporary Survey, 19, Tel Aviv 1995, S. 562 (569f.); Abullahi A. An-Na'im / Francis M. Deng, a.a.O. (Fn. 137), S. 211; Scott Peterson, a.a.O, (Fn. 149), S. 206; Hanspeter Mattes, Der Sudan: Vielfältige Dimensionen der Entwicklung, in: Hanspeter Mattes / Sigrid Faath [Hrsg.], Wuquf 7-8, Hamburg 1993, S. 11 (42,); anschaulich auch ders., Ibid., S. 125 ff., insb. Schaubild 2 'Spaltung der SPLA seit 1991' auf S. 139. 
Beide Parteien müssen also noch den Nachweis antreten, die erfolgversprechenden Vereinbarungen für ihren Teil tatsächlich umzusetzen. ${ }^{151}$ Ergänzend stehen sie aber auch gemeinsam in der Verantwortung, sich auf dem Weg zu einem umfassenden, vor allem jedoch nachhaltigen Frieden, der festgelegten Mechanismen zu bedienen, um die ansatzweise aufgezeigten Hindernisse zu bewältigen. Erforderliche Änderungen der Übergangsverfassung, welche Regelungen des Friedensvertrages betreffen, können nämlich nur mit Zustimmung der SPLM und der Zentralregierung in Khartum in Angriff genommen werden. $^{152}$

\section{Schlussfolgerung}

Bei aller Kritik ist festzustellen, dass die Aussichten einer friedlichen Lösung besser denn je stehen. Um diese positiven Ansätze nicht wieder eine ungenutzte Chance werden zu lassen, muss seitens der internationalen Gemeinschaft weiterhin der multilaterale Lösungsansatz oberste Priorität genießen. Wichtig ist dabei aber auch das Eingeständnis so genannter „Geberländer“, zuweilen die Komplexität der regionalen Gegebenheiten nicht adäquat einschätzen zu können und den regional initiierten Konfliktlösungs- und Entwicklungsansätzen nicht ausreichend Handlungsspielraum zu gewähren. Aufgrund der insgesamt hohen Konfliktintensität am Horn von Afrika (i.w.S.) und sich dort stetig wiederholender humanitärer Katastrophen, erfordert diese politisch labile Subregion des afrikanischen Kontinents weiterhin erhöhte Aufmerksamkeit und besondere Unterstützung in der Friedenssicherung und Konfliktprävention. Für den Sudan bedeutet dies konkret, dass der bisher bilateral strukturierte Prozess zwischen der Zentralregierung in Khartum und der SPLM unbedingt ausgeweitet werden muss. Das wird sich sicherlich noch über viele Jahre hinziehen. Maßgeblich ist dabei jedoch letztlich die anhaltenden Kontinuität der Bemühungen und die Nachhaltigkeit eines tatsächlich umfassenden Friedens im Sudan und damit die Stabilität der Region.

151 So auch Wolfgang Heinrich, a.a.O. (Fn. 9), S. 1054.

152 Power Sharing Agreement, Part II (Institutions at the National Level), 2.2.7.2. 